\title{
On the mechanism of stomatocyte-echinocyte transformations of red blood cells: experiment and theoretical model
}

\author{
K.D. Tachev ${ }^{\text {a }}$, K.D. Danov ${ }^{\text {b }}$, P.A. Kralchevsky ${ }^{\text {b,* }}$ \\ a Department of Functional Biology, Faculty of Natural Sciences, University of Shumen, 9712 Shumen, Bulgaria \\ ${ }^{\mathrm{b}}$ Laboratory of Chemical Physics and Engineering, Faculty of Chemistry, University of Sofia, 1164 Sofia, Bulgaria
}

Received 4 December 2003

\begin{abstract}
This study represents an attempt to achieve a better understanding of the stomatocyte-echinocyte transition in the shape of red blood cells. We determined experimentally the index of cell shape at various ionic strengths and osmolarities for native and trypsin-treated human erythrocytes. For every given composition of the outer phase, we calculated the ionic strength in the cells and the transmembrane electric potential using a known theoretical model. Next, we described theoretically the electric double layers formed on both sides of the cell membrane, and derived expressions for the tensions of the two membrane leaflets. Taking into account that the cell-shape index depends on the tension difference between the two leaflets, we fitted the experimental data with the constructed physicochemical model. The model, which agrees well with the experiment, indicates that the tension difference between the two leaflets is governed by the different adsorptions of counterions at the two membrane surfaces, rather than by the direct contribution of the electric double layers to the membrane tension. Thus, with the rise of the ionic strength, the counterion adsorption increases stronger at the outer leaflet, whose stretching surface pressure becomes greater, and whose area expands relative to that of the inner leaflet. Hence, there is no contradiction between the bilayer-couple hypothesis and the electric double layer theory, if the latter is upgraded to account for the effect of counterion-adsorption on the membrane tension. The developed quantitative model can be applied to predict the shape index of cells upon a stomatocyte-discocyte-echinocyte transformation at varying composition of the outer medium.
\end{abstract}

(C) 2004 Elsevier B.V. All rights reserved.

Keywords: Counterion adsorption; Bilayer-couple hypothesis; Echinocyte; Electric double layer; Erythrocyte shape; Index of cell shape; Membrane tension; Red blood cells; Stomatocyte

\section{Introduction}

In many studies, it has been established that the normal red blood cells (discocytes) undergo shape transformations toward stomatocytes or echinocytes (Fig. 1) upon variation of the electrolyte concentration [1-7], increase of the medium's $\mathrm{pH}$ [7-12], addition of amphiphiles and other agents [1,13-18], and changes in temperature [19,20]. A general correlation was found between the erythrocyte shape and the transmembrane electric potential [21-25]. The effect of $\mathrm{pH}$ and other agents could be also attributed to their influence on the transmembrane potential [25]. Detailed reviews, different viewpoints and discussions on the molecular origin of the observed phenomena can be found in

* Corresponding author. Tel.: +359-2-9625310; fax: +359-2-9625643.

E-mail address: pk@lcpe.uni-sofia.bg (P.A. Kralchevsky).
[7,12,18,24-26]. Some authors have established correlations between the stomatocyte-echinocyte transition and the effect of additives on the conformation of the anion-exchange protein band 3, including inhibition of the anion transport effectuated by this protein [27-30].

A basis for a quantitative theory of the stomatocyte-echinocyte transformation is provided by the bilayer-couple hypothesis (model), proposed by Sheetz and Singer [15], and Evans [31]. According to this hypothesis, any factor, which leads to expansion of the outer membrane leaflet relative to the inner one, produces a tendency to form convex structures, as the echinocytic spicules. Conversely, any expansion of the inner leaflet, relative to the outer one, favors the formation of cavities (stomatocytosis) to accommodate the extra area. In terms of the membrane mechanics [32], the area (and tension) difference between the two leaflets leads to the appearance of a large spontaneous curvature of the lipid bilayer. In an impressive study, Lim et al. [33] applied a mechanical 


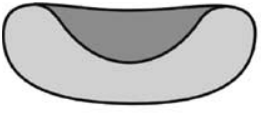

(a) stomatocyte

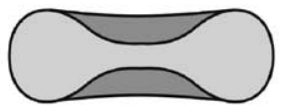

(b) discocyte

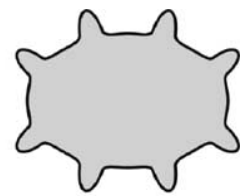

(c) echinocyte
Fig. 1. Sketch of erythrocyte shapes: (a) stomatocyte with a "moon-like" cross-section; (b) discocyte with a biconcave-disc shape; (c) echinocyte covered with protruding "spicules". With the increase of the medium's ionic strength, at fixed osmolarity, a red blood cell undergoes shape transformations in the sequence stomatocyte $\rightarrow$ discocyte $\rightarrow$ echinocyte.

model to calculate the minimum energy shapes at increasing area difference between the two leaflets; they obtained shapes in the sequence stomatocyte-discocyte-echinocyte (Fig. 1) in agreement with the bilayer-couple hypothesis and with the experimentally observed shapes.

The next step toward a full understanding of the cell-shape transformations should be to relate the area difference between the leaflets with the changes in the medium's ionic strength and the transmembrane potential. Lange et al. [34] and Grebe et al. [35] have considered the electrostatic repulsion between charged residues in membrane surface as an expansive force, which can create the area difference between the leaflets. As this electrostatic repulsion can be quantitatively predicted by the electric double layer theory [36], the coupling of the latter with a membrane-mechanical model, like that in [33], would lead to the construction of a complete quantitative theory of the stomatocyte-echinocyte transformation. Unfortunately, it seems that the experimental observations contradict the predictions of the double-layer theory: with the rise of the outer medium's ionic strength the experiment shows a shifting of the stomatocytes toward echinocytes [3], whereas exactly the opposite trend follows from the double layer theory insofar as the repulsion between the charges at the outer membrane surface should be screened by the increased electrolyte concentration; consequently the area of the outer leaflet should diminish [7].

In fact, the increase of the ionic strength affects the repulsion between the surface charges in two ways: firstly, by the aforementioned direct screening of the Coulombic interactions by the electrolyte, and secondly, by adsorption (binding) of counterions at the negatively charged surface groups, which neutralizes their electric charge and diminishes the magnitude of the net surface charge density. Moreover, the binding of counterions, which is characterized by certain adsorption energy, reduces the interfacial (membrane) tension in a similar way, as the adsorption of amphiphilic molecules lowers the surface tension of the respective solution. The effect of counterion adsorption turned out to be essential for the development of a quantitative theory of micellization [37-39] and surface tension of ionic surfactant solutions [40-42]. The latter effects may have a considerable influence on the tensions and areas of the membrane leaflets, and therefrom-on the shape of the cell.
Our purpose in the present article is to compare experimental data for the shape index of erythrocytes with a detailed physicochemical model of electrolyte influence on the membrane tension. We followed a quantitative approach that, in principle, could allow us to estimate and compare the significance of the involved factors.

The paper is organized as follows. Section 2 describes the experimental procedures. In Section 3, we report data for the cell shape index at various ionic strengths and osmolarities of the outer medium. Section 4 is devoted to the full set of equations determining the variation of the net surface charge and electric field due to changes in the ionic strength. Section 5 gives a theoretical description of the effect of ionic strength on the membrane tension. Finally, in Section 6 we compare theory and experiment, present and discuss the fits of the experimental data, and compare the relative importance of the involved physicochemical factors.

Let us mention in advance, that the direct effect of the electric double layers on the membrane tension turns out to be negligible because of the high experimental ionic strength which, on the other hand, leads to a significant adsorption of counterions that engenders a tension and area difference between the two leaflets. The latter is large enough to produce erythrocyte shape transformations, in agreement with the bilayer-couple hypothesis and the experiment.

\section{Experimental system and procedures}

\subsection{Solutions used in the experiments}

To prepare Tris-buffer solutions, we used Trizma base and Trizma HCl, chemically pure, products of Sigma (St. Louis, USA). The other chemicals, $\mathrm{NaCl}, \mathrm{NaH}_{2} \mathrm{PO}_{4}, \mathrm{Na}_{2} \mathrm{HPO}_{4}$, and $\mathrm{MgSO}_{4}$ were of analytical grade. The samples of erythrocytes were preserved in an isotonic Tris-buffer with $\mathrm{pH}=7.4$ at $25^{\circ} \mathrm{C}$. This buffer contained $0.129 \mathrm{M} \mathrm{NaCl}$, 0.0147 M Trizma $\mathrm{HCl}$ and 0.0023 M Tizma base.

The working solutions of various ionic strength and osmolarity were obtained by addition of the necessary amounts of salts $\left(\mathrm{NaCl}, \mathrm{MgSO}_{4}\right)$, sucrose and Tris-buffer. The concentration of the latter was always adjusted to be $17 \mathrm{mM}(0.0147 \mathrm{M}$ Trizma $\mathrm{HCl}$ and $0.0023 \mathrm{M}$ Tizma base). The experimental concentrations of $\mathrm{NaCl}, \mathrm{MgSO}_{4}$ and sucrose are listed in Table 1. As usual, the ionic strength is $I=1 / 2 \sum_{i}\left(Z_{i}^{2} c_{i}\right)$, where $Z_{i}$ and $c_{i}$ are the valence and bulk concentration of the $i$ th ion; the summation is carried out over all ionic species in the respective aqueous phase.

The used trypsin, product of Merck (Darmstadt, Germany), had an activity of $2000 \mathrm{E} / \mathrm{g}$. The working isotonic solutions contained $15 \mu \mathrm{g} / \mathrm{ml}$ trypsin, $0.129 \mathrm{M} \mathrm{NaCl}$ and $0.017 \mathrm{M}$ Tris-buffer.

All solutions were prepared with doubly-distilled water, which had a specific electro-resistance of about $1 \mathrm{M} \Omega \mathrm{cm}$. 
Table 1

Ionic strength and osmolarity of the working solutions, and the used concentrations of salts and sucrose

\begin{tabular}{cccc}
\hline Ionic strength $(\mathrm{mM})$ & $\mathrm{NaCl}(\mathrm{mM})$ & $\mathrm{MgSO}_{4}(\mathrm{mM})$ & $\mathrm{C}_{12} \mathrm{H}_{22} \mathrm{O}_{11}(\mathrm{mM})$ \\
\hline Osmolarity 146 mOsm & & & \\
45.01 & 23.63 & 1.67 & 63.70 \\
94.98 & 49.44 & 7.71 & 0 \\
144.99 & 32.77 & 24.38 & 0 \\
200.85 & 14.15 & 43.00 & 0 \\
243.30 & 0 & 57.15 & 0 \\
Osmolarity 156 mOsm & & & \\
45.00 & 30.30 & 0 & 63.70 \\
95.00 & 56.10 & 6.05 & 0 \\
145.01 & 39.43 & 22.72 & 0 \\
200.00 & 21.10 & 41.05 & 0 \\
263.30 & 0 & 62.15 & 0 \\
& & & \\
Osmolarity 290 mOsm & & & \\
45.00 & 30.30 & 0 & 197.70 \\
95.00 & 70.30 & 2.50 & 112.70 \\
143.85 & 99.15 & 7.50 & 45.00 \\
203.85 & 109.15 & 20.00 & 0 \\
301.35 & 76.65 & 52.50 & 0 \\
401.85 & 43.15 & 86.00 & 0 \\
503.85 & 9.15 & 120.00 & \\
\hline
\end{tabular}

\subsection{Procedures with the red blood cells}

Human erythrocytes from blood group " 0 ”, factor $\mathrm{Rh}(-)$, were used. They were isolated from venous blood samples stabilized with heparin (product of Spofa, Prague). The samples were prepared in the following way. The blood was centrifugated at $1000 \times g$ for $10 \mathrm{~min}$. The plasma was removed by aspiration. The sedimented cells were "washed" by resuspending in an isotonic buffer solution added in an amount, which was three times greater than the volume of the sediment. Next, the new suspension was centrifugated at the same conditions. This washing procedure was repeated three times. Finally, we added isotonic buffer to the erythrocyte sediment to obtain a stock suspension of volume fraction (hematocrit) 50\%. The stock suspension can be preserved up to 3 days at $4{ }^{\circ} \mathrm{C}$.

The working erythrocyte suspensions were prepared as follows. In a test tube we poured $5 \mathrm{ml}$ of the respective working solution (see Table 1) and $0.2 \mathrm{ml}$ of the stock erythrocyte suspension. The mixture was homogenized by shaking. After 1 min the suspension was loaded in the working Bürker chamber.

\subsection{Enzymatic treatment of the cell membrane}

By enzymatic treatment it is possible to detach hydrophilic portions of the glycoproteins at the outer surface of the cell membrane. After $[43,44]$, in our experiments we applied the enzyme trypsin, which hydrolyzes bonds (not necessarily peptide) in which the carboxyl groups belong to the amino acids lysin and arginin. The trypsin-treatment leads to a decrease in the thickness of the cell glycocalyx, and to a reduc- tion in the negative electric charge at the outer membrane surface.

The used procedure was the following. First, we prepared a $15 \mu \mathrm{g} / \mathrm{ml}$ solution of trypsin in isotonic buffer. The "washed" red blood cells were resuspended in the latter solution, at a hematocrit of $50 \%$, and were then incubated at $37^{\circ} \mathrm{C}$ for $1 \mathrm{~h}$. After that, the erythrocytes were centrifuged and washed three times by isotonic buffer. Finally, the stock suspension was obtained by resuspending of the cells in a buffer solution to achieve again a hematocrit of $50 \%$.

\subsection{Microscopic observations}

To observe the erythrocytes, we used a microscope NU-2 (Carl Zeiss, Jena) with an objective of magnification $40 \times$ and numerical aperture 0.80 . The source of illumination was a halogen lamp, $100 \mathrm{~W}$. A video camera was mounted to observe and record the microscopic images.

In the observation field of the microscope a large square $(200 \mu \mathrm{m} \times 200 \mu \mathrm{m})$ sector of the Bürker chamber was seen. For each erythrocyte sample, two different square sectors were processed. Each of these sectors was observed and recorded twice: 10 and $30 \mathrm{~min}$ after the contact of the investigated red blood cells with the respective working solution of a given ionic strength and osmolarity. By superimposing the video frames taken at the 10th and 30th minute, we were able to detect the changes in the shape and size of each separate cell.

\subsection{Processing of the recorded images}

With the help of the computer program "Mocha" (Jandel Scientific) for digital image analysis, we assigned labels with shape indices to the observed erythrocytes. Nine shapes were considered in accordance with the following classification [7,14]: biconcave disc (shape index 0); three types of stomatocytes (indexes $-1,-2$ and -3 ) to spherostomatocyte (index -4); and three degrees of echinocytes (indexes 1,2 and 3) to spheroechinocyte (index 4). At least 150 cells were classified and labeled for each processed frame. Erythrocytes of various indexes are present in a given sample. For each frame, the indexes were recorded in a computer and then the mean-arithmetic index of cell shape was computed.

\section{Experimental results and discussion}

\subsection{Role of the ionic strength and osmolarity}

As shown in Table 1, in our experiments we worked at three different fixed values of the osmolarity: $290 \mathrm{mOsm}$ (isosmotic solution), and two hypotonic solutions: at 156 and $146 \mathrm{mOsm}$. In the hypotonic solutions, the erythrocytes are markedly swollen [44]. In these solutions, we observed cells of increased volume, but still some cells exhibited stomatocyte and echinocyte features. For each fixed osmolarity, the 
ionic strength of the ambient solution, $I_{2}$, was varied from 45 to $500 \mathrm{mM}$ for the isosmotic solutions. For the hypotonic solutions, there was an upper boundary for $I_{2}$, stemming from the fixed osmolarity. For that reason, the greatest ionic strength was $I_{2}=263 \mathrm{mM}$ for $156 \mathrm{mOsm}$, and $I_{2}=$ $243 \mathrm{mM}$ for $146 \mathrm{mOsm}$.

Initially, almost all of the red blood cells in the plasma had the discocyte shape. Upon immersion of the cells in the

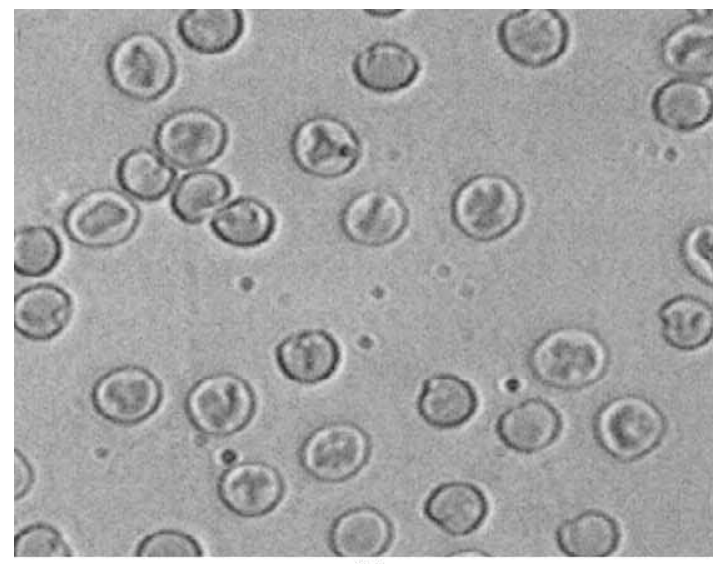

(a)

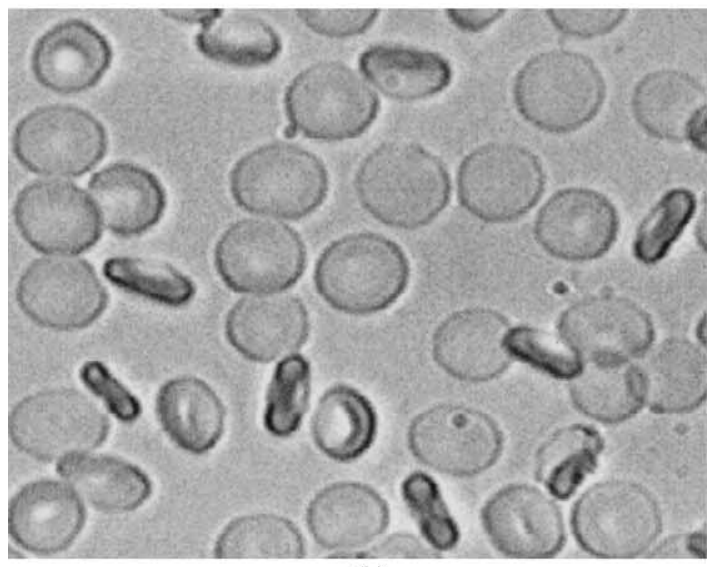

(b)

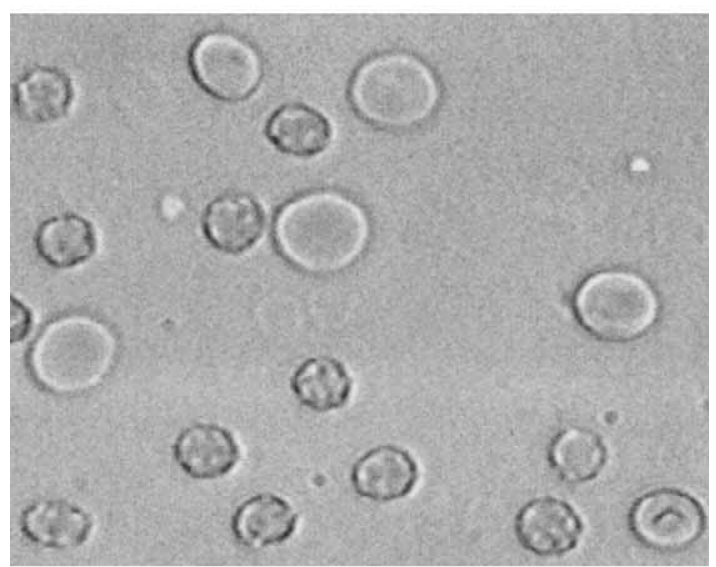

(c)

Fig. 2. Shapes of erythrocytes in solutions of osmolarity $290 \mathrm{mOsm}$ at three different ionic strengths of the outer medium: (a) $I_{2}=46 \mathrm{mM}$; (b) $I_{2}=96 \mathrm{mM}$; (c) $I_{2}=300 \mathrm{mM}$. working solutions (see Table 1), their shape undergoes quick changes. Fig. 2 shows photos of erythrocytes in isosmotic solutions $(290 \mathrm{mOsm})$ at three different ionic strengths. One sees that at the lowest ionic strength, $I_{2}=45 \mathrm{mM}$, the stomatocyte shapes prevail, whereas at the highest ionic strength, $I_{2}=300 \mathrm{mM}$, most of the red blood cells are echinocytes. Note that initially, before their dispersion in the working solution, the cells were discocytes. As seen in Fig. 2, after the first $10 \mathrm{~min}$, the cells had practically reached their equilibrium shape for the respective solution.

The above tendency is confirmed by the statistics for the shape index. Fig. 3 shows plots of the erythrocyte mean-arithmetic shape index versus the ionic strength of the solution at two fixed values of the osmolarity: 290 and $146 \mathrm{mOsm}$. One sees that at the lower ionic strengths, the shape index is negative; in other words, the predominant shape is stomatocyte. At the higher ionic strengths, the shape index is positive, which evidences echinocyte shapes. This
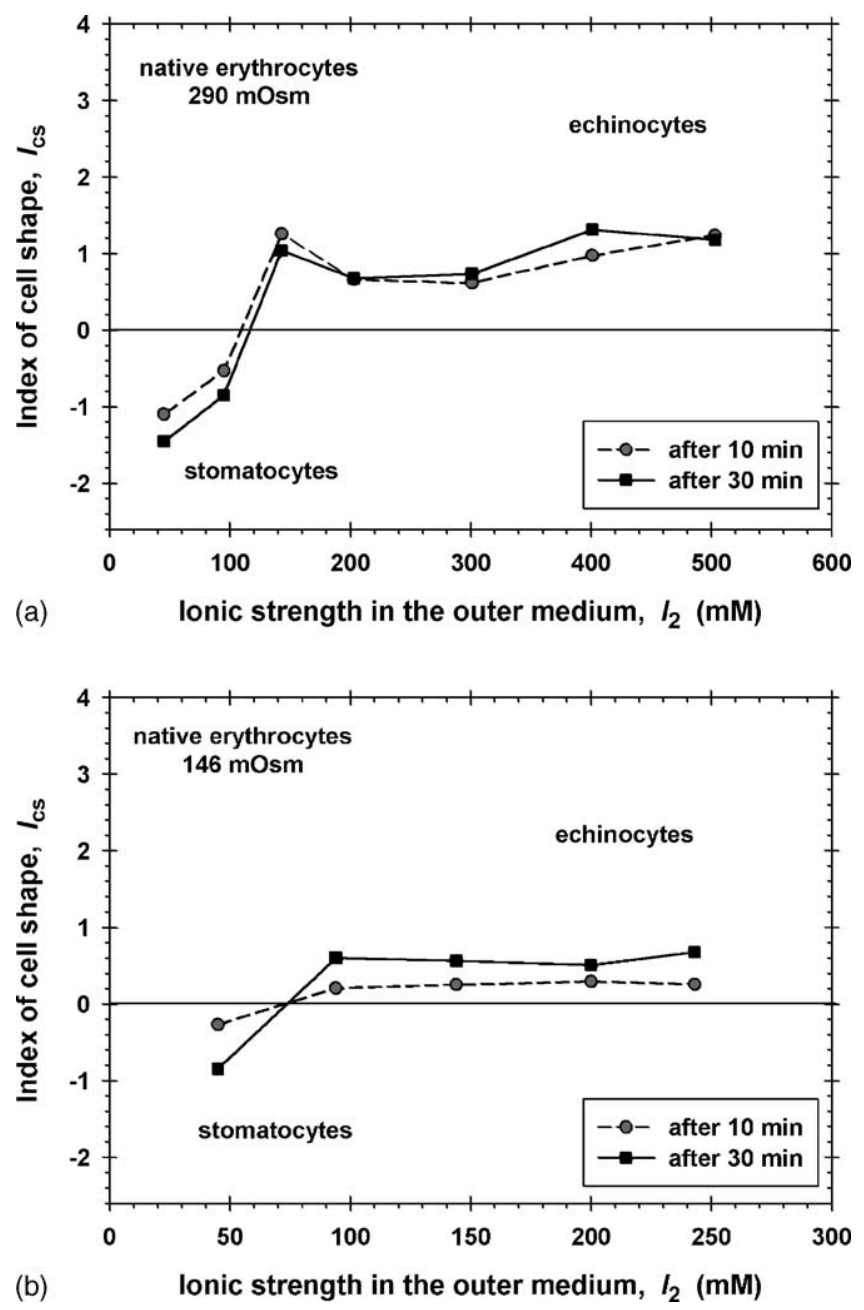

Fig. 3. Plot of the experimental index of cell shape, $I_{\mathrm{cs}}$, vs. the ionic strength of the outer medium, $I_{2}$, for erythrocytes, which have not been treated with trypsin: osmolarity (a) $290 \mathrm{mOsm}$; (b) $146 \mathrm{mOsm}$. The values of $I_{\mathrm{cs}}$, measured 10 and $30 \mathrm{~min}$ after the immersion of the cells in the respective solutions, are compared. 

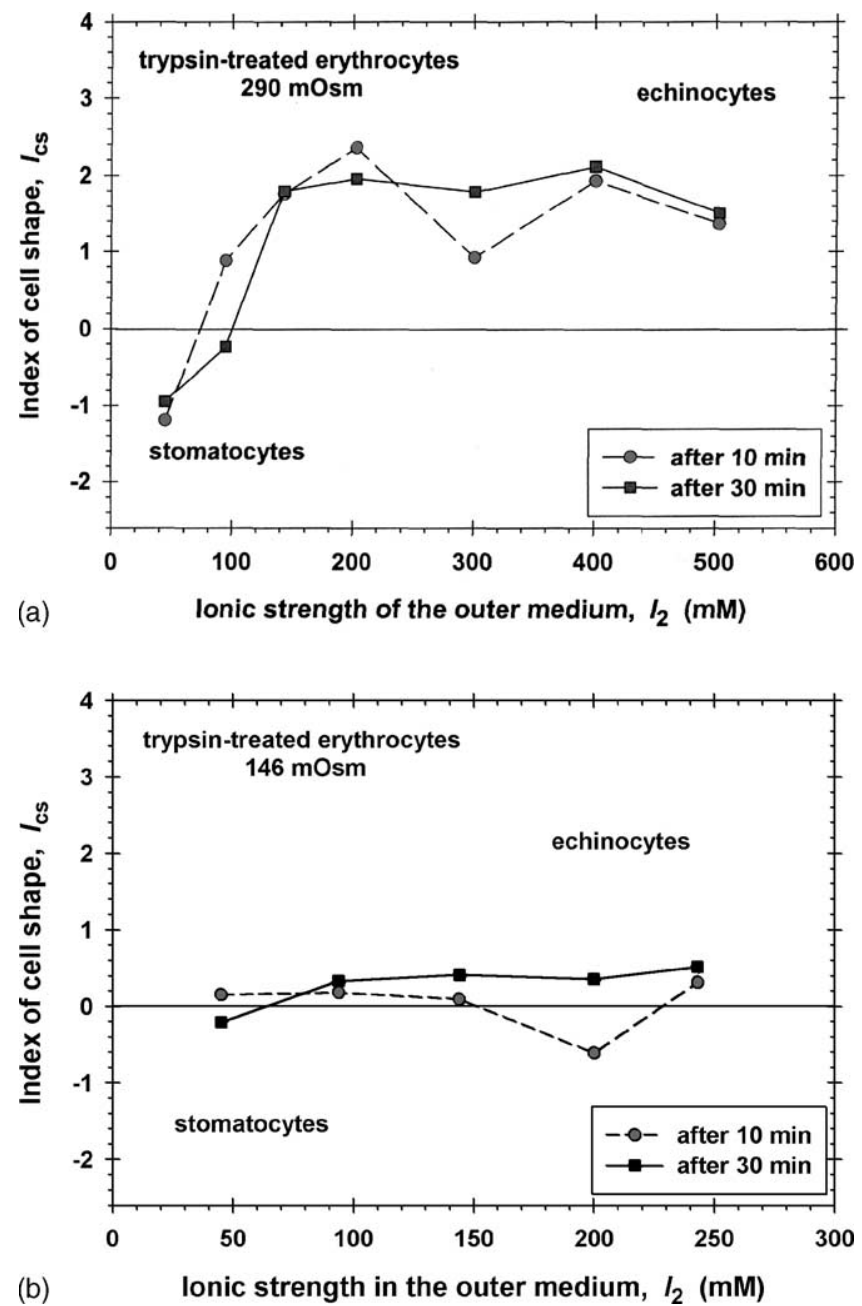

Fig. 4. Plot of the experimental index of cell shape, $I_{\mathrm{cs}}$, vs. the ionic strength of the outer medium, $I_{2}$, for erythrocytes treated with trypsin: osmolarity (a) $290 \mathrm{mOsm}$; (b) $146 \mathrm{mOsm}$. The values of $I_{\mathrm{cs}}$, measured 10 and $30 \mathrm{~min}$ after the immersion of the cells in the respective solutions, are compared.

tendency is the best pronounced at osmolarity $290 \mathrm{mOsm}$ : one sees a relatively steep transition from stomatocyte to echinocyte shapes at ionic strength of about $100 \mathrm{mM}$, Fig. 3a. For the hypotonic solution, $146 \mathrm{mOsm}$, the amplitude of the deviation from discocyte shape (shape index 0 ) is relatively smaller, Fig. $3 \mathrm{~b}$ (the results for $156 \mathrm{mOsm}$, which look very similar to those for $146 \mathrm{mOsm}$, are not shown in Fig. 4). Our experimental results are in agreement with data by other authors, e.g. [3,24].

\subsection{Results for trypsin-treated erythrocytes}

As mentioned in Section 2.3, the trypsin hydrolyzes a part of the molecular fragments in the glycocalyx thus reducing its thickness and the number of ionizable groups at the outer membrane surface. This is expected to affect the tension of the outer leaflet of the membrane and to influence the shape index of the cells [7]. Our data (Fig. 4)

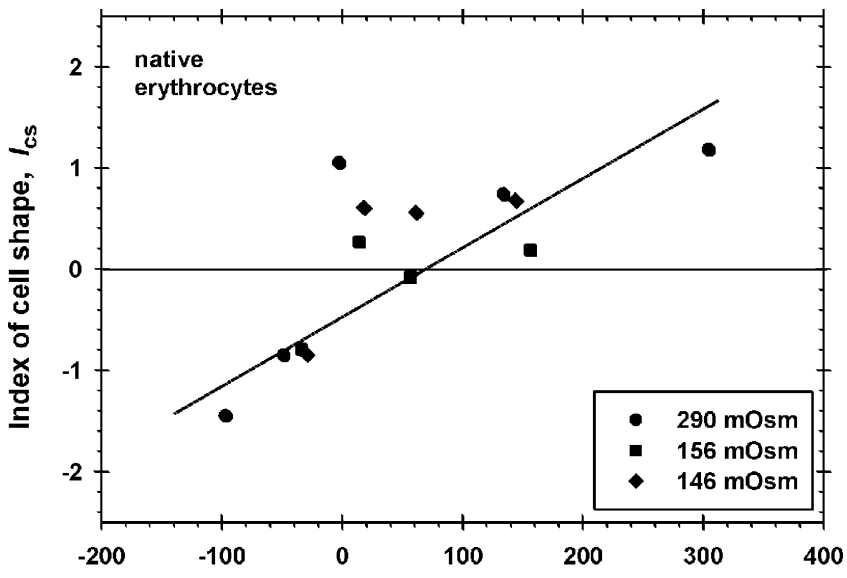

Difference between the ionic strengths, $\Delta I=I_{2}-I_{1}(\mathrm{mM})$

Fig. 5. Plot of the experimental cell-shape index, $I_{\mathrm{cs}}$, vs. the difference between the ionic strengths in the outer and inner phases, $\Delta I \equiv I_{2}-I_{1}$; data from Table 2. The line is a guide to the eye.

show that the shape index of the trypsin-treated red blood cells is slightly shifted toward echinocytes. The effect is better pronounced for osmolarity $290 \mathrm{mOsm}$; compare Figs. 3a and $4 \mathrm{a}$. For the hypotonic solutions, the differences between the treated and non-treated cells are smaller, in the framework of the experimental accuracy, see Figs. $4 \mathrm{~b}$ and $5 \mathrm{~b}$. In Sections 4 and 5, we develop a model accounting for the effects of surface charge density and counterion binding on the membrane tension. This model provides a quantitative interpretation of our experimental data for erythrocytes with and without trypsin-treatment.

\subsection{Transmembrane potential and shape index}

As mentioned earlier, Glaser and co-workers [23-25] established that the shape transformation correlates with the transmembrane potential and cell shape. To check whether our data indicates the presence of such a correlation, we determined the ionic concentrations in the membrane interior and the transmembrane potential.

The problem about the passive and active transport of ions across the membrane of human red blood cell is well studied. In our calculations, we used a computer program developed on the basis of the integrated red cell model of transmembrane transport by Lew and Bookchin [45], which has been upgraded to account for some recently found effects of ionic and specific transport [46]. This program involves the kinetic parameters of most of the known transport systems and enables one to calculate the ionic concentrations, the $\mathrm{pH}$ and the water contents inside the cell for each given composition and $\mathrm{pH}$ in the outer medium. We applied this program to the working solutions, whose compositions are specified in Table 1. Thus we computed the ionic strength, $I_{1}$, inside the cell, and the transmembrane electric potential, $\Delta \psi$, which characterizes the state of Donnan equilibrium between the cell interior and exterior. The sign of $\Delta \psi$ is 
Table 2

Parameters for native erythrocytes (non-treated with trypsin): Experimental ionic strength in the outer medium, $I_{2}$, and cell shape index $I_{\mathrm{cs}}$

\begin{tabular}{|c|c|c|c|c|c|c|c|}
\hline$I_{2}(\mathrm{mM})$ & $I_{1}(\mathrm{mM})$ & $\Delta \Phi$ & $I_{\mathrm{cs}}$ & $\Phi_{\mathrm{s} 1}$ & $\Phi_{\mathrm{s} 2}$ & $\gamma(\mathrm{mN} / \mathrm{m})$ & $\Delta \gamma(\mathrm{mN} / \mathrm{m})$ \\
\hline \multicolumn{8}{|c|}{ Cell at standard physiological conditions } \\
\hline 151.8 & 151.5 & -0.463 & 0 & -0.462 & -0.146 & 0 & 0 \\
\hline \multicolumn{8}{|c|}{ Cells at osmolarity $290 \mathrm{mOsm}$} \\
\hline 46.24 & 143.1 & 0.644 & -1.45 & 0.638 & -0.486 & 0.347 & -0.247 \\
\hline 95.79 & 144.3 & 0.005 & -0.85 & 0.003 & -0.242 & 0.189 & -0.096 \\
\hline 144.2 & 146.5 & -0.333 & 1.05 & -0.333 & -0.154 & 0.051 & 0.015 \\
\hline 300.5 & 166.5 & -0.613 & 0.74 & -0.612 & -0.062 & -0.360 & 0.164 \\
\hline 500.0 & 195.3 & -0.757 & 1.18 & -0.755 & -0.032 & -0.748 & 0.225 \\
\hline \multicolumn{8}{|c|}{ Cells at osmolarity $156 \mathrm{mOsm}$} \\
\hline 46.62 & 80.46 & 0.072 & -0.79 & 0.066 & -0.486 & 0.940 & 0.348 \\
\hline 97.54 & 83.34 & -0.456 & 0.27 & -0.457 & -0.239 & 0.750 & 0.476 \\
\hline 148.0 & 91.24 & -0.551 & -0.08 & -0.551 & -0.150 & 0.529 & 0.512 \\
\hline 266.1 & 109.7 & -0.705 & 0.19 & -0.703 & -0.073 & 0.119 & 0.544 \\
\hline \multicolumn{8}{|c|}{ Cells at osmolarity $146 \mathrm{mOsm}$} \\
\hline 47.58 & 76.98 & 0.183 & -0.84 & 0.177 & -0.477 & 0.981 & 0.396 \\
\hline 97.26 & 79.11 & -0.404 & 0.61 & -0.406 & -0.240 & 0.804 & 0.528 \\
\hline 149.3 & 87.77 & -0.495 & 0.56 & -0.495 & -0.148 & 0.566 & 0.554 \\
\hline 247.5 & 103.2 & -0.644 & 0.68 & -0.643 & -0.080 & 0.212 & 0.577 \\
\hline
\end{tabular}

Ionic strength inside the cell, $I_{1}$, and dimensionless transmembrane potential, $\Delta \Phi$, calculated using the model from [45,46]. Dimensionless inner and outer membrane surface potentials, $\Phi_{\mathrm{s} 1}$ and $\Phi_{\mathrm{s} 2}$, membrane tension, $\gamma$, and difference between the tensions of the inner and outer membrane leaflet, $\Delta \gamma$, calculated in Sections 4-6. In the computations, we have set $\gamma=\Delta \gamma=0$ for the cells under standard physiological conditions.

defined in such a way that a positive $\Delta \psi$ means that the electric potential in the cell interior is greater than that in the bulk of the outer medium.

The first four columns of Table 2 show, respectively, the experimental ionic strength in the outer medium, $I_{2}$, the computed ionic strength inside the cell, $I_{1}$, the dimensionless transmembrane potential

$$
\Delta \Phi=\frac{e \Delta \psi}{k T}
$$

and values of the cell shape index, $I_{\mathrm{cs}}$, measured $30 \mathrm{~min}$ after immersion of the cells in the working solution, see Fig. 2. In Eq. (3.1), $e$ is the electronic charge, $k$ is the Boltzmann constant and $T$ is the temperature $\left(25^{\circ} \mathrm{C}\right.$ in our experiments); all electric potentials are scaled with $k T / e=25.75 \mathrm{mV}$. The rest of the columns in Table 2 represent calculated values of the dimensionless surface potentials and membrane tensions, see Section 6 for details.

Our data in Table 2 show that $I_{\mathrm{cs}}$ really correlates with $\Delta \Phi$, in consonance with the results of Glaser and co-workers

Table 3

Parameters for trypsin-treated erythrocytes

\begin{tabular}{|c|c|c|c|c|c|c|c|}
\hline$I_{2}(\mathrm{mM})$ & $I_{1}(\mathrm{mM})$ & $\Delta \Phi$ & $I_{\mathrm{cS}}$ & $\Phi_{\mathrm{s} 1}$ & $\Phi_{\mathrm{s} 2}$ & $\gamma(\mathrm{mN} / \mathrm{m})$ & $\Delta \gamma(\mathrm{mN} / \mathrm{m})$ \\
\hline \multicolumn{8}{|c|}{ Cell at standard physiological conditions } \\
\hline 151.8 & 151.5 & -0.463 & 0 & -0.462 & -0.076 & 0 & 0 \\
\hline \multicolumn{8}{|c|}{ Cells at osmolarity $290 \mathrm{mOsm}$} \\
\hline 46.24 & 143.1 & 0.644 & -0.95 & 0.639 & -0.287 & 0.311 & -0.207 \\
\hline 95.79 & 144.3 & 0.005 & -0.24 & 0.003 & -0.130 & 0.164 & -0.069 \\
\hline 144.2 & 146.5 & -0.333 & 1.77 & -0.332 & -0.080 & 0.048 & 0.020 \\
\hline 300.5 & 166.5 & -0.613 & 1.70 & -0.612 & -0.031 & -0.296 & 0.101 \\
\hline 500.0 & 195.3 & -0.757 & 1.49 & -0.755 & -0.016 & -0.620 & 0.098 \\
\hline \multicolumn{8}{|c|}{ Cells at osmolarity $156 \mathrm{mOsm}$} \\
\hline 46.62 & 80.46 & 0.072 & -0.57 & 0.068 & -0.288 & 0.903 & 0.389 \\
\hline 97.54 & 83.34 & -0.456 & 0.15 & -0.456 & -0.130 & 0.725 & 0.503 \\
\hline 148.0 & 91.24 & -0.551 & 0.48 & -0.550 & -0.078 & 0.528 & 0.515 \\
\hline 266.1 & 109.7 & -0.705 & 0.39 & -0.703 & -0.037 & 0.170 & 0.494 \\
\hline \multicolumn{8}{|c|}{ Cells at osmolarity $146 \mathrm{mOsm}$} \\
\hline 47.58 & 76.98 & 0.183 & -0.21 & 0.178 & -0.281 & 0.945 & 0.436 \\
\hline 97.26 & 79.11 & -0.404 & 0.33 & -0.405 & -0.130 & 0.779 & 0.555 \\
\hline 149.3 & 87.77 & -0.495 & 0.31 & -0.495 & -0.077 & 0.565 & 0.557 \\
\hline 247.5 & 103.2 & -0.644 & 0.52 & -0.643 & -0.040 & 0.256 & 0.535 \\
\hline
\end{tabular}

The meaning of the parameters $I_{2}, I_{1}, \Delta \Phi, I_{\mathrm{cs}}, \Phi_{\mathrm{s} 1}, \Phi_{\mathrm{s} 2}, \gamma$ and $\Delta \gamma$ is the same as in Table 2. 
[23-25]. In particular, $I_{\mathrm{cs}}$ and $\Delta \Phi$ change sign almost simultaneously; stomatocytes and echinocytes are predominant for $\Delta \Phi>0$ and $\Delta \Phi<0$, respectively (Table 2).

The data in Table 2 indicates also a correlation between $I_{\mathrm{cs}}$ and the difference between the ionic strengths in the two phases, $\Delta I \equiv I_{2}-I_{1}$, which is illustrated in Fig. 5. Dyscocytes $\left(I_{\mathrm{cs}}=0\right)$ are formed around $\Delta I=0$; the stomatocytes and echinocytes prevail, respectively, for $I_{2}<I_{1}$ and $I_{2}>I_{1}$.

Table 3 is similar to Table 2, but it presents data for the trypsin-treated erythrocytes (Fig. 4), rather than for the native ones (Fig. 3). Such a treatment is supposed to affect the glycocalyx, but it is not expected to influence the Donnan equilibrium between the intra- and extracellural phases. Then, for the same $I_{2}$ and osmolarity, the calculated values of $I_{1}$ and $\Delta \Phi$ are the same for native and trypsin-treated cells (Tables 2 and 3). On the other hand, the experiment gives different values for $I_{\mathrm{cs}}$ in Tables 2 and 3, which is to be considered as a result of the trypsin-treatment.

The correlation of $I_{\mathrm{cs}}$ with $\Delta \Phi$ and $\Delta I$ implies that the shape transformation stomatocyte-echinocyte is somehow related to the electric properties of the cell membrane. Our purpose in the next section is to quantify this relationship on the basis of a detailed model of the electric field distribution in the vicinity of the cell membrane. This model takes into account the electric charge density at the inner and outer membrane surfaces; the formation of electric double layers inside and outside the membrane; the non-zero electric field across the membrane; the binding of counterions to the two surfaces of the membrane; the electrostatic and ion-adsorption contributions to the tensions of the two membrane leaflets, etc. Our purpose is to identify the factor(s) governing the stomatocyte-echinocyte transformation, and to better understand the mechanism of its occurrence.

\section{Electric field distribution}

\subsection{Expressions for the electric potential}

Negatively charged ionizable groups are present at the inner and outer surfaces of the membrane of an erythrocyte $[47,48]$. It is known that the surface charges give rise to the formation of electric double layers [36,49,50], which have to be present on both sides of the cell membrane. The expected distribution of the electric potential across the membrane, $\psi(z)$, is sketched in Fig. 6, the $z$-axis being normal to the membrane. It is convenient to denote by $\psi_{1}(z), \psi_{\mathrm{m}}(z)$, and $\psi_{2}(z)$, respectively, the electric potential in the inner double layer $(z<-h)$, that in the hydrocarbon interior of the membrane $(-h<z<h)$, and that in the outer double layer (Fig. 6). Here and hereafter, the indexes 1 and 2 will refer to the aqueous phases, respectively, inside and outside the cell, and the index " $\mathrm{m}$ "- - to the hydrocarbon core of the membrane. The surface electric potentials on the two
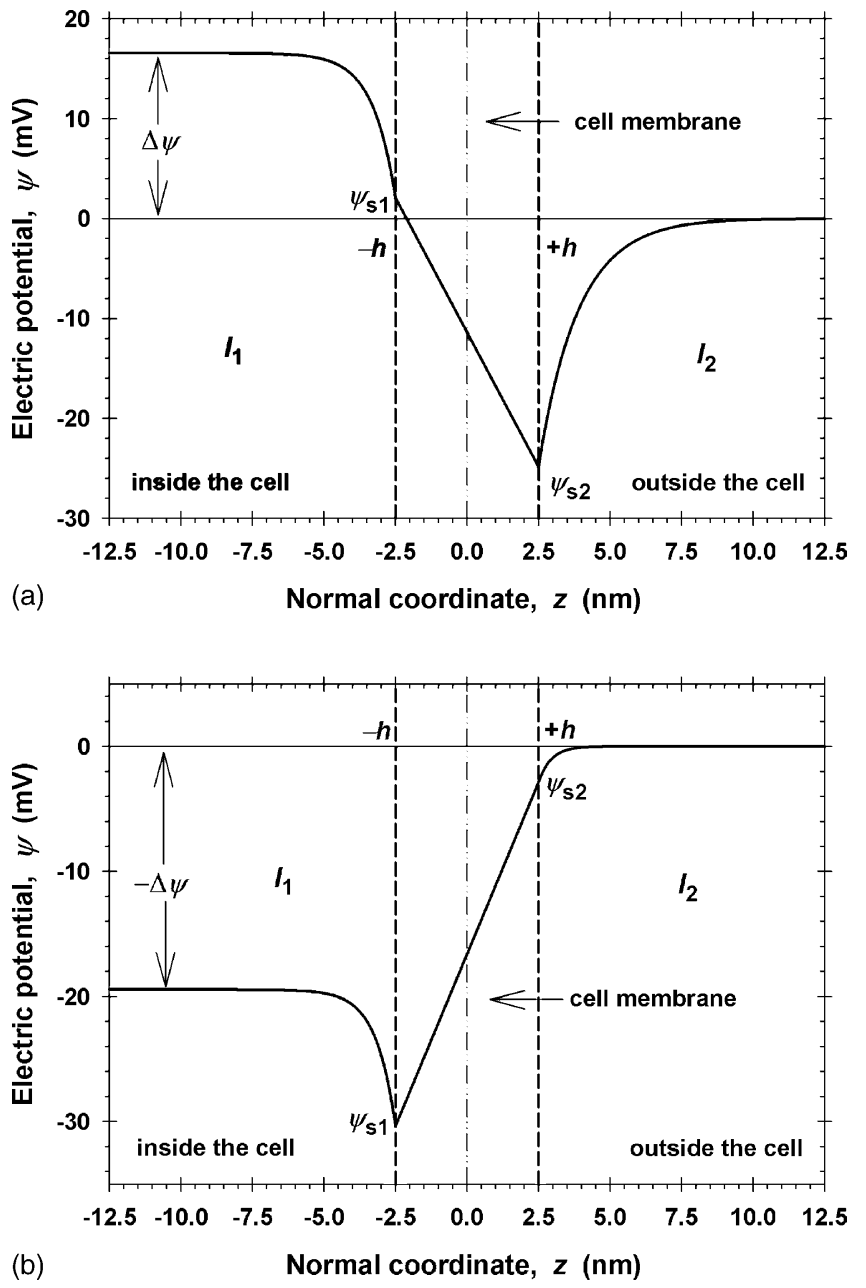

Fig. 6. Expected profile of the electric potential, $\psi(z)$, across the cell membrane; the latter is situated at $-h<z<+h$; $I_{1}$ and $I_{2}$ are the ionic strengths inside and outside the cell, respectively; $\psi_{\mathrm{s} 1}$ and $\psi_{\mathrm{s} 2}$ are the surface potentials at the inner and outer membrane surfaces; $\Delta \psi$ is the transmembrane potential: (a) $\Delta \psi$ is positive for a lower $I_{2}$; (b) $\Delta \psi$ is negative for a higher $I_{2}$, see Table 2 .

sides of the membrane will be denoted by $\psi_{\mathrm{s} 1}=\psi(-h)$ and $\psi_{\mathrm{s} 2}=\psi(h)$. Furthermore, it is convenient to introduce dimensionless electric potentials:

$\begin{array}{ll}\Phi_{1}=\frac{e \psi_{1}}{k T}, & \Phi_{\mathrm{s} 1}=\frac{e \psi_{\mathrm{s} 1}}{k T}, \\ \Phi_{2}=\frac{e \psi_{2}}{k T}, & \Phi_{\mathrm{s} 2}=\frac{e \psi_{\mathrm{s} 2}}{k T}\end{array}$

$(k T / e=25.75 \mathrm{mV})$. The Debye screening parameters for the two aqueous phases, $\kappa_{1}$ and $\kappa_{2}$, are related to the respective ionic strengths, $I_{1}$ and $I_{2}[36,49,50]$ :

$\kappa_{1}^{2} \equiv \frac{8 \pi e^{2} I_{1}}{\varepsilon_{\mathrm{w}} k T}, \quad \kappa_{2}^{2} \equiv \frac{8 \pi e^{2} I_{2}}{\varepsilon_{\mathrm{w}} k T}$

$\varepsilon_{\mathrm{W}}$ is the dielectric constant of water. For the distribution of the electric potential in the two aqueous phases, we will use 
a known equation from the double layer theory:

$$
\begin{aligned}
& \tanh \left(\frac{\Phi_{1}-\Delta \Phi}{4}\right) \\
& \quad=\tanh \left(\frac{\Phi_{\mathrm{s} 1}-\Delta \Phi}{4}\right) \exp \left[\kappa_{1}(z+h)\right] \quad(z \leq-h)
\end{aligned}
$$

$\tanh \left(\frac{\Phi_{2}}{4}\right)=\tanh \left(\frac{\Phi_{\mathrm{s} 2}}{4}\right) \exp \left[-\kappa_{2}(z-h)\right] \quad(z \geq h)$

see e.g. [36], or Eq. (1.65) in [51]. Note that for $z \rightarrow \infty$, Eq. (4.4) yields $\Phi_{2} \rightarrow 0$, that is the potential in the bulk of the outer water phase is set zero, by definition. On the other hand, for $z \rightarrow-\infty$, Eq. (4.3) gives $\Phi_{1} \rightarrow \Delta \Phi$, the latter being the value of the dimensionless transmembrane potential, see Fig. 6 and Eq. (3.1). The bulk solutions are assumed uniform and electroneutral, as usual in the double layer theory.

Using Eqs. (4.3) and (4.4), we have made the following simplifying assumptions. Firstly, the intracellular and extracellulal water phases are assumed to be solutions of symmetric electrolytes, having the same ionic strengths, $I_{1}$ or $I_{2}$, as the real phases, see Tables 2 and 3. Secondly, the aforementioned model solutions are treated as ideal, that is the respective ionic activity coefficients are set equal to 1 . The latter assumption is not expect to lead to a considerable mistake, insofar as the modeling of the intracellular phase as a diluted electrolyte solution is found to give correct predictions about the concentrations of ions due to inorganic electrolytes, such as $\mathrm{K}^{+}, \mathrm{Na}^{+}, \mathrm{Cl}^{-}$, etc. [52].

The membrane interior is modeled as an oily film of thickness $2 h$, where $h$ is approximately equal to the average chain-length of the lipid molecules. In this film, electric charges are absent, and consequently, the Poisson equation reduces to $\mathrm{d}^{2} \Phi_{\mathrm{m}} / \mathrm{d} z^{2}=0$, which implies that $\Phi_{\mathrm{m}}$ is a linear function of $z$ :

$\Phi_{\mathrm{m}}=\Phi_{\mathrm{s} 1} \frac{h-z}{2 h}+\Phi_{\mathrm{s} 2} \frac{z+h}{2 h} \quad(-h \geq z \leq h)$

see Fig. 6 and Eq. (4.1). To relate the surface potentials, $\Phi_{\mathrm{s} 1}$, and $\Phi_{\mathrm{s} 2}$, with the respective surface electric charge densities, $\sigma_{\mathrm{s} 1}$, and $\sigma_{\mathrm{s} 2}$, we have to apply the respective standard electrostatic boundary conditions [53]:

$\varepsilon_{\mathrm{w}} \frac{\mathrm{d} \Phi_{1}}{\mathrm{~d} z}-\varepsilon_{\mathrm{m}} \frac{\mathrm{d} \Phi_{\mathrm{m}}}{\mathrm{d} z}=\frac{4 \pi e}{k T} \sigma_{\mathrm{s} 1} \quad(z=-h)$

$\varepsilon_{\mathrm{w}} \frac{\mathrm{d} \Phi_{2}}{\mathrm{~d} z}-\varepsilon_{\mathrm{m}} \frac{\mathrm{d} \Phi_{\mathrm{m}}}{\mathrm{d} z}=-\frac{4 \pi e}{k T} \sigma_{\mathrm{s} 2} \quad(z=h)$

where $\varepsilon_{\mathrm{m}}$ is the dielectric constant of the hydrocarbon medium in the core of the cell membrane. The substitution of Eqs. (4.3)-(4.5) into Eqs. (4.6) and (4.7), yields:

$\sinh \left(\frac{\Phi_{\mathrm{s} 1}-\Delta \Phi}{2}\right)-\frac{\varepsilon_{\mathrm{m}}}{\varepsilon_{\mathrm{w}}} \frac{\Phi_{\mathrm{s} 2}-\Phi_{\mathrm{s} 1}}{4 h \kappa_{1}}=\frac{2 \pi e \sigma_{\mathrm{s} 1}}{k T \varepsilon_{\mathrm{w}} \kappa_{1}}$ $\sinh \left(\frac{\Phi_{\mathrm{s} 2}}{2}\right)+\frac{\varepsilon_{\mathrm{m}}}{\varepsilon_{\mathrm{w}}} \frac{\Phi_{\mathrm{s} 2}-\Phi_{\mathrm{s} 1}}{4 h \kappa_{2}}=\frac{2 \pi e \sigma_{\mathrm{s} 2}}{k T \varepsilon_{\mathrm{w}} \kappa_{2}}$

To get a full set of equation, we need additional relations between the surface charge and potential, which represent equations describing the adsorption of counterions at the membrane surfaces.

\subsection{Adsorption of counterions at the membrane surfaces}

The theoretical analysis of experimental data has shown that adsorption (binding) of counterions takes place at the boundary of an ionic solution. As mentioned earlier, this has been established for adsorption monolayers [40-42] and surfactant micelles [37-39]. For the system under consideration, we can expect that the negatively charged ionizable groups on the two membrane surfaces serve as adsorption sites for positively charged cations $\left(\mathrm{K}^{+}, \mathrm{Na}^{+}\right.$, etc.). To describe the counterion adsorption, we will employ the Stern model [54-56], which has been found to compare very well with the experiment [57-59].

Let $\Gamma_{1}^{(1)}$ and $\Gamma_{1}^{(2)}$ be the surface density of negatively charged groups on the inner and outer membrane surfaces, respectively. $\Gamma_{1}^{(1)}$ is determined by the surface density of the headgroups of the lipid phosphatidylserine in the inner membrane leaflet [47], whereas $\Gamma_{1}^{(2)}$ is mostly due to the sialic acid residues in the glycocalyx [48]. Furthermore, let $\Gamma_{2}^{(1)}$ and $\Gamma_{2}^{(2)}$ be the surface density of the adsorbed counterions, respectively, at the inner and outer membrane surfaces. The Stern model [54] gives the following expressions for $\Gamma_{2}^{(1)}$ and $\Gamma_{2}^{(2)}$ :

$\frac{\Gamma_{2}^{(1)}}{\Gamma_{1}^{(1)}}=\frac{K_{1} I_{1} \exp \left(\Delta \Phi-\Phi_{\mathrm{s} 1}\right)}{1+K_{1} I_{1} \exp \left(\Delta \Phi-\Phi_{\mathrm{s} 1}\right)}$

$\frac{\Gamma_{2}^{(2)}}{\Gamma_{1}^{(2)}}=\frac{K_{2} I_{2} \exp \left(-\Phi_{\mathrm{s} 2}\right)}{1+K_{2} I_{2} \exp \left(-\Phi_{\mathrm{s} 2}\right)}$

where $K_{1}$ and $K_{2}$ denote the Stern adsorption constants. The net surface charge is proportional to the difference between the surface densitties of negative surface groups and positive adsorbed counterions:

$\sigma_{\mathrm{s} 1}=-e\left(\Gamma_{1}^{(1)}-\Gamma_{2}^{(1)}\right)=\frac{-e \Gamma_{1}^{(1)}}{1+K_{1} I_{1} \exp \left(\Delta \Phi-\Phi_{\mathrm{s} 1}\right)}$

$\sigma_{\mathrm{s} 2}=-e\left(\Gamma_{1}^{(2)}-\Gamma_{2}^{(2)}\right)=\frac{-e \Gamma_{1}^{(2)}}{1+K_{2} I_{2} \exp \left(-\Phi_{\mathrm{s} 2}\right)}$

where $\Gamma_{2}^{(1)}$ and $\Gamma_{2}^{(2)}$ have been substituted from Eqs. (4.10) and (4.11). To simplify our model, in Eqs. (4.10)-(4.13) we have assumed that the adsorbing cations are monovalent (like $\mathrm{K}^{+}, \mathrm{Na}^{+}$). The presence of some divalent counterions $\left(\mathrm{Ca}^{2+}, \mathrm{Mg}^{2+}\right)$ may lead to determining of greater effective 
values of the constants $K_{1}$ and $K_{2}$ from the fits of experimental data.

The Stern adsorption constants can be expressed in the form $[41,56]$ :

$$
K_{i}=\frac{\delta_{i}}{\Gamma_{1}^{(i)}} \exp \left(\frac{\Delta \mu^{(i)}}{k T}\right), \quad i=1,2
$$

where $\delta_{i}$ is the diameter of the hydrated counterion and $\Delta \mu^{(i)}$ characterizes the adsorption energy, that is the difference between the standard chemical potentials of the ion in the bulk and in the interfacial adsorption layer. In our model calculations, we will use $\delta_{i}=0.7 \mathrm{~nm}$, which is approximately equal to the diameter of the hydrated $\mathrm{K}^{+}$and $\mathrm{Na}^{+}$ions [50]. To estimate $\Gamma_{1}^{(1)}$, we take into account that the phosphatidylserine constitutes $12.5 \%$ of the lipids in the inner membrane leaflet [47], and that the average area per lipid in the membrane is about $0.5 \mathrm{~nm}^{2}$. Thus we obtain $\Gamma_{1}^{(1)}=0.25 \mathrm{~nm}^{-2}$; in other words, the area per negatively charged headgroup is $\left[\Gamma_{1}^{(1)}\right]^{-1}=4 \mathrm{~nm}^{2}$ at the inner leaflet. On the outer membrane surface, the surface charge, due to the sialic, acid is estimated to be $0.0198 \mathrm{C} / \mathrm{m}^{2}$ [48], which corresponds to $\Gamma_{1}^{(2)}=0.124 \mathrm{~nm}^{-2}$ (the negative charge on both inner and outer membrane surfaces is due mostly to carboxylic groups, which are expected to be completely dissociated at the working $\mathrm{pH}=7.4$ ). The latter values of $\Gamma_{1}^{(1)}$ and $\Gamma_{1}^{(2)}$ are used in our computations. Because the adsorption energies in Eq. (4.14), $\Delta \mu^{(1)}$ and $\Delta \mu^{(2)}$, are not known, they will be determined as adjustable parameters from the data fits.

At present, the literature data indicate the existence of weak and strong binding of counterions, as assumed by Kalinin and Radke [40]. For the weak binding, the adsorption energy of a monovalent counterion is typically $\Delta \mu^{(i)} \approx 2 k T$, say $1.64 k T$ for $\mathrm{Na}^{+}$ions at sulfate headgroups [39,41,57]. In this case, the binding energy can be attributed to a residual electrostatic attraction between oppositely charged counterions and surface groups, which is due to the discreteness of the surface charge (not taken into account by the continuum double layer theory) [39]. For the strong binding, the adsorption energy is $\Delta \mu^{(i)} \approx 9 k T$, see e.g. Table 4 in [59], which is close to the energy of formation of a weak hydrogen bond between water molecules, $20-25 \mathrm{~kJ} / \mathrm{mol}$, that is $8-10 \mathrm{kT}$ [60]. For example, such a bond might be $-\mathrm{COO}^{-} \cdots \mathrm{H}-\mathrm{O}-\mathrm{H}$, where the carboxilic group belongs to the head of phosphatidylserine, while the water molecule belongs to the hydration shell of a $\mathrm{K}^{+}$ion (or some other cation present in the cell). The fits of our data (Section 6) indicate that such a bond could be really formed.

\subsection{The full set of equations}

Eqs. (4.8), (4.9), (4.12) and (4.13) form a full set for determining the four quantities, $\Phi_{\mathrm{s} 1}, \Phi_{\mathrm{s} 2}, \sigma_{\mathrm{s} 1}$ and $\sigma_{\mathrm{s} 2}$, for every given ionic strength $I_{2}$, supposedly all other parameters are known (note that $I_{1}$ and $\Delta \Phi$ are given in Tables 2 and 3 for each given value of $I_{2}$ ). In particular, substituting $\sigma_{\mathrm{s} 1}$ from Eq. (4.12) into Eq. (4.8), we derive:

$$
\begin{aligned}
\Phi_{\mathrm{s} 2}= & \Phi_{\mathrm{s} 1}+4 h \kappa_{1} \frac{\varepsilon_{\mathrm{w}}}{\varepsilon_{\mathrm{m}}}\left[\sinh \left(\frac{\Phi_{\mathrm{s} 1}-\Delta \Phi}{2}\right)\right. \\
& \left.+\frac{2 \pi e^{2} \Gamma_{1}^{(1)}}{k T \varepsilon_{\mathrm{w}} \kappa_{1}} \frac{1}{1+K_{1} I_{1} \exp \left(\Delta \Phi-\Phi_{\mathrm{s} 1}\right)}\right]
\end{aligned}
$$

Likewise, substituting $\sigma_{\mathrm{s} 2}$ from Eq. (4.13) into Eq. (4.9), we obtain the equation $F\left(\Phi_{\mathrm{s} 1}\right)=0$, where

$$
\begin{aligned}
F\left(\Phi_{\mathrm{s} 1}\right)= & \sinh \left(\frac{\Phi_{\mathrm{s} 2}}{2}\right)+\frac{\varepsilon_{\mathrm{m}}}{\varepsilon_{\mathrm{w}}} \frac{\Phi_{\mathrm{s} 2}-\Phi_{\mathrm{s} 1}}{4 h \kappa_{2}} \\
& +\frac{2 \pi e^{2} \Gamma_{1}^{(2)}}{k T \varepsilon_{\mathrm{w}} \kappa_{2}} \frac{1}{1+K_{2} I_{2} \exp \left(-\Phi_{\mathrm{s} 2}\right)}
\end{aligned}
$$

Eq. (4.15) determines $\Phi_{\mathrm{s} 2}=\Phi_{\mathrm{s} 2}\left(\Phi_{\mathrm{s} 1}\right)$. Then, Eq. (4.16) defines $F$ as a function of $\Phi_{\mathrm{s} 1}$, alone. Moreover, $F\left(\Phi_{\mathrm{s} 1}\right)$ is a monotonic function, and consequently, the equation $F\left(\Phi_{\mathrm{s} 1}\right)=0$ has a single root for $\Phi_{\mathrm{s} 1}$. In our computations, this root was determined numerically, by means of the bisection method. After that, Eqs. (4.15), (4.12) and (4.13) give, respectively, $\Phi_{\mathrm{s} 2}, \sigma_{\mathrm{s} 1}$ and $\sigma_{\mathrm{s} 2}$.

\section{Effect of ionic strength on membrane tension}

The tension of the cell membrane, $\gamma$, can be expressed in the form:

$\gamma=\tilde{\gamma}_{\mathrm{el}}+\tilde{\gamma}_{\mathrm{ad}}+\tilde{\gamma}_{0}$

where $\tilde{\gamma}_{\mathrm{el}}$ is the direct contribution of the electric field due to the two adjacent diffuse electric double layers, see Fig. 6; $\tilde{\gamma}_{\mathrm{ad}}$ is a contribution from the adsorption of counterions on the two surfaces of the membrane, see Section 4.2; $\tilde{\gamma}_{0}$ accounts for all other contributions to the membrane tension. Eq. (5.1) is analogous to the expression used for the interfacial tension of an ionic surfactant solution, see e.g. [41,51,55, 61].

The ionic strength can affect the tension of the cell membrane, $\gamma$, at least in two ways. Firstly, the diffuse electric double layer shrinks with the increase of the ionic strength and this effect influences the value of $\tilde{\gamma}_{\text {el }}$ [7]. Secondly, the rise of ionic strength enhances the adsorption of counterions, which will lead to a variation of $\tilde{\gamma}_{\text {ad }}$. Our aim below is to quantify these effects.

There is a general expression for $\tilde{\gamma}_{\mathrm{el}}$, which follows from the known Bakker formula [62,63]:

$\tilde{\gamma}_{\mathrm{el}}=\int_{-\infty}^{+\infty}\left[P_{N}^{(\mathrm{el})}-P_{T}^{(\mathrm{el})}\right] \mathrm{d} z$

where $P_{N}^{(\mathrm{el})}$ and $P_{T}^{(\mathrm{el})}$ are the normal and tangential component of the Maxwell electrostatic pressure tensor. Using known expressions for $P_{N}^{(\mathrm{el})}$ and $P_{T}^{(\mathrm{el})}[41,51,53,61,64]$, one 
can bring Eq. (5.2) into the form:

$\tilde{\gamma}_{\mathrm{el}}=-\int_{-\infty}^{+\infty} \frac{\varepsilon}{4 \pi}\left(\frac{\mathrm{d} \psi}{\mathrm{d} z}\right)^{2} \mathrm{~d} z=-\frac{k T}{4 \pi e} \int_{-\infty}^{+\infty} \varepsilon\left(\frac{\mathrm{d} \Phi}{\mathrm{d} z}\right)^{2} \mathrm{~d} z$

Representing the latter integral as a sum of three terms, we obtain:

$\tilde{\gamma}_{\mathrm{el}}=\tilde{\gamma}_{1, \mathrm{el}}+\tilde{\gamma}_{\mathrm{m}, \mathrm{el}}+\tilde{\gamma}_{2, \mathrm{el}}$

where

$\tilde{\gamma}_{1, \mathrm{el}}=-\frac{\varepsilon_{\mathrm{W}} k T}{4 \pi e} \int_{-\infty}^{-h}\left(\frac{\mathrm{d} \Phi_{1}}{\mathrm{~d} z}\right)^{2} \mathrm{~d} z$

$\tilde{\gamma}_{\mathrm{m}, \mathrm{el}}=-\frac{\varepsilon_{\mathrm{m}} k T}{4 \pi e} \int_{-h}^{+h}\left(\frac{\mathrm{d} \Phi_{\mathrm{m}}}{\mathrm{d} z}\right)^{2} \mathrm{~d} z$

$\tilde{\gamma}_{2, \text { el }}=-\frac{\varepsilon_{\mathrm{w}} k T}{4 \pi e} \int_{+h}^{+\infty}\left(\frac{\mathrm{d} \Phi_{2}}{\mathrm{~d} z}\right)^{2} \mathrm{~d} z$

Next, we express the derivatives $\mathrm{d} \Phi / \mathrm{d} z$ from Eqs. (4.3)-(4.5), and substitute the results into the latter integrals. After some transformations, we derive

$\tilde{\gamma}_{1, \mathrm{el}}=-8 k T \frac{I_{1}}{\kappa_{1}}\left[\cosh \left(\frac{\Delta \Phi-\Phi_{\mathrm{s} 1}}{2}\right)-1\right]$

$\tilde{\gamma}_{2, \mathrm{el}}=-8 k T \frac{I_{2}}{\kappa_{2}}\left[\cosh \left(\frac{\Phi_{\mathrm{s} 2}}{2}\right)-1\right]$

$\tilde{\gamma}_{\mathrm{m}, \mathrm{el}}=-\frac{\varepsilon_{\mathrm{m}}(k T)^{2}}{8 \pi h e^{2}}\left(\Phi_{\mathrm{s} 2}-\Phi_{\mathrm{s} 1}\right)^{2}$

Expressions analogous to Eqs. (5.6) and (5.7) are well known in the theory of the electric double layer $[41,51,55,61]$.

Since the Stern isotherms, Eqs. (4.10) and (4.11), correspond to a Langmuirian type of adsorption, it is self-consistent to express $\tilde{\gamma}_{\text {ad }}$ by means of the equation of Szyszkowski-Langmuir, see e.g. [51,65,66]:

$\tilde{\gamma}_{\text {ad }}=\tilde{\gamma}_{1, \text { ad }}+\tilde{\gamma}_{2, \text { ad }}$

where

$\tilde{\gamma}_{i, \text { ad }}=-k T \Gamma_{1}^{(i)} \ln \left(1-\frac{\Gamma_{2}^{(i)}}{\Gamma_{1}^{(i)}}\right), \quad i=1,2$

In Eq. (5.10), we have supposed that the additive constant in the Szyszkowski-Langmuir equation is included in $\tilde{\gamma}_{0}$. Having in mind Eqs. (5.4) and (5.9), we bring Eq. (5.1) into the form:

$\gamma=\tilde{\gamma}_{1, \mathrm{el}}+\tilde{\gamma}_{2, \mathrm{el}}+\tilde{\gamma}_{\mathrm{m}, \mathrm{el}}+\tilde{\gamma}_{1, \mathrm{ad}}+\tilde{\gamma}_{2, \mathrm{ad}}+\tilde{\gamma}_{0}$

Now, only the additive parameter $\tilde{\gamma}_{0}$ is unknown. We can determine $\tilde{\gamma}_{0}$ taking into account that an erythrocyte at standard physiological conditions (a discocyte), is expected to be in a tension-free state, $\gamma=0$. For the latter case, Eq. (5.11) can be written in the form:

$0=\tilde{\gamma}_{1, \mathrm{el}}^{(\mathrm{st})}+\tilde{\gamma}_{2, \mathrm{el}}^{(\mathrm{st})}+\tilde{\gamma}_{\mathrm{m}, \mathrm{el}}^{(\mathrm{st})}+\tilde{\gamma}_{1, \mathrm{ad}}^{(\mathrm{st})}+\tilde{\gamma}_{2, \mathrm{ad}}^{(\mathrm{st})}+\tilde{\gamma}_{0}$ where the superscript '( $\mathrm{st})$ ' denotes properties of a cell under standard physiological conditions, see the first rows of Tables 2 and 3. $\tilde{\gamma}_{0}$ is expected to be independent of the solution's ionic strength. Moreover, in a first approximation, $\tilde{\gamma}_{0}$ can be considered as independent of osmolarity, except for osmolarities which are very close to the point of cell lysis $[45,46]$. Subtracting Eq. (5.12) from Eq. (5.11) and introducing the notations

$\gamma_{i, \mathrm{el}}=\tilde{\gamma}_{i, \mathrm{el}}-\tilde{\gamma}_{i, \mathrm{el}}^{(\mathrm{st})}, \quad \gamma_{\mathrm{m}, \mathrm{el}}=\tilde{\gamma}_{\mathrm{m}, \mathrm{el}}-\tilde{\gamma}_{\mathrm{m}, \mathrm{el}}^{(\mathrm{st})}$,

$\gamma_{i, \mathrm{ad}}=\tilde{\gamma}_{i, \mathrm{ad}}-\tilde{\gamma}_{i, \mathrm{ad}}^{(\mathrm{st})}$

$(i=1,2)$ we get the following final expression for the membrane tension:

$\gamma=\gamma_{1, \mathrm{el}}+\gamma_{2, \mathrm{el}}+\gamma_{\mathrm{m}, \mathrm{el}}+\gamma_{1, \mathrm{ad}}+\gamma_{2, \mathrm{ad}}$

where, in view of Eqs. (5.6)-(5.8), (5.10) and (5.13), we have

$$
\begin{aligned}
\gamma_{1, \mathrm{el}}= & 8 k T\left\{\frac{I_{1}^{(\mathrm{st})}}{\kappa_{1}^{(\mathrm{st})}}\left[\cosh \left(\frac{\Delta \Phi^{(\mathrm{st})}-\Phi_{\mathrm{s} 1}^{(\mathrm{st})}}{2}\right)-1\right]\right. \\
& \left.-\frac{I_{1}}{\kappa_{1}}\left[\cosh \left(\frac{\Delta \Phi-\Phi_{\mathrm{s} 1}}{2}\right)-1\right]\right\} \\
\gamma_{2, \mathrm{el}}= & 8 k T\left\{\frac{I_{2}^{(\mathrm{st})}}{\kappa_{2}^{(\mathrm{st})}}\left[\cosh \left(\frac{\Phi_{\mathrm{s} 2}^{(\mathrm{st})}}{2}\right)-1\right]\right. \\
& \left.-\frac{I_{2}}{\kappa_{2}}\left[\cosh \left(\frac{\Phi_{\mathrm{s} 2}}{2}\right)-1\right]\right\} \\
\gamma_{\mathrm{m}, \mathrm{el}}= & \frac{\varepsilon_{\mathrm{m}}(k T)^{2}}{8 \pi h e^{2}}\left[\left(\Phi_{\mathrm{s} 2}^{(\mathrm{st})}-\Phi_{\mathrm{s} 1}^{(\mathrm{st})}\right)^{2}-\left(\Phi_{\mathrm{s} 2}-\Phi_{\mathrm{s} 1}\right)^{2}\right] \\
\gamma_{i, \text { ad }}= & k T \Gamma_{1}^{(i)}\left[\ln \left(1-\frac{\Gamma_{2}^{(i, \mathrm{st})}}{\Gamma_{1}^{(i)}}\right)-\ln \left(1-\frac{\Gamma_{2}^{(i)}}{\Gamma_{1}^{(i)}}\right)\right]
\end{aligned}
$$

$(i=1,2)$. For the computations, it is more convenient to substitute $\Gamma_{2}^{(i)} / \Gamma_{1}^{(i)}$ from Eqs. (4.10) and (4.11) into Eq. (5.18); thus we obtain:

$\gamma_{1, \mathrm{ad}}=k T \Gamma_{1}^{(1)} \ln \left[\frac{1+K_{1} I_{1} \exp \left(\Delta \Phi-\Phi_{\mathrm{s} 1}\right)}{1+K_{1} I_{1, \mathrm{st}} \exp \left(\Delta \Phi^{(\mathrm{st})}-\Phi_{\mathrm{s} 1}^{(\mathrm{st})}\right)}\right]$

$\gamma_{2, \mathrm{ad}}=k T \Gamma_{1}^{(2)} \ln \left[\frac{1+K_{2} I_{2} \exp \left(-\Phi_{\mathrm{s} 2}\right)}{1+K_{2} I_{2}^{(\mathrm{st})} \exp \left(-\Phi_{\mathrm{s} 2}^{(\mathrm{st})}\right)}\right]$

Eqs. (5.15)-(5.20), enable one to calculate the contribution of the diffuse electric double layers to the tensions of the inner and outer membrane leaflets, $\gamma_{1, \text { el }}$ and $\gamma_{2, \text { el }}$, as well as the contribution of the counterion adsorption to the tensions of the two leaflets, $\gamma_{1, \text { ad }}$ and $\gamma_{2, \text { ad }}$. Eqs. (4.5) and (5.5) imply 
that $\gamma_{\mathrm{m}, \mathrm{el}}$ gives equal contributions to the tensions of the two membrane leaflets, which therefore become:

$\gamma_{1}=\gamma_{1, \text { ad }}+\gamma_{1, \mathrm{el}}+\frac{1}{2} \gamma_{\mathrm{m}, \mathrm{el}}$
$\gamma_{2}=\gamma_{2, \mathrm{ad}}+\gamma_{2, \mathrm{el}}+\frac{1}{2} \gamma_{\mathrm{m}, \mathrm{el}}$

Consequently, the difference between the tensions of the two leaflets of the membrane can be expressed in the form:

$\Delta \gamma=\gamma_{1}-\gamma_{2}=\Delta \gamma_{\mathrm{ad}}+\Delta \gamma_{\mathrm{el}}=\pi_{\mathrm{s}}^{(\mathrm{out})}-\pi_{\mathrm{s}}^{(\mathrm{in})}$

where $\pi_{\mathrm{s}}^{(\text {out })}=-\gamma_{2}$ and $\pi_{\mathrm{s}}^{(\mathrm{in})}=-\gamma_{1}$ are the surface pressures of the outer and inner leaflets, and

$\Delta \gamma_{\mathrm{ad}}=\gamma_{1, \mathrm{ad}}-\gamma_{2, \mathrm{ad}}, \quad \Delta \gamma_{\mathrm{el}}=\gamma_{1, \mathrm{el}}-\gamma_{2, \mathrm{el}}$

In summary, the equations derived in the present section allow us to calculate the total membrane tension, $\gamma$, the difference between the tensions of the two leaflets, $\Delta \gamma$ (see the last columns of Tables 2 and 3), and other related quantities, which will be utilized in the next section to interprete the observed transformation stomatocyte-echinocyte.

\section{Comparison of theory and experiment}

\subsection{Relation between cell shape and membrane tension}

If the variation of some factor, say the ionic strength, leads to an increase of the difference between the surface pressures of the two membrane leaflets, $\pi_{\mathrm{s}}^{(\text {out })}-\pi_{\mathrm{s}}^{(\mathrm{in})}$, this will lead to an expansion of the outer leaflet relative to the inner one, and will produce an area difference, $\Delta A_{0}$, between the two leaflets. As we are dealing with small area variations, we can apply the Hooke's law of elasticity to relate stress and strain:

$$
\frac{\Delta A_{0}}{A}=\frac{1}{K_{\mathrm{s}}}\left(\pi_{\mathrm{s}}^{(\text {out })}-\pi_{\mathrm{s}}^{(\text {in })}\right)=\frac{1}{K_{\mathrm{s}}} \Delta \gamma
$$

where $A$ is the total area of the membrane, $K_{\mathrm{S}}$ is the stretching elastic modulus of one membrane leaflet; Eq. (5.23) has been also used.

In accordance with the bilayer-couple hypothesis [15,31-33], any increase of $\Delta A_{0}$ produces a tendency to form convex structures on the cell surface (e.g. echinocytic spicules) to accommodate the extra area of the outer leaflet; conversely, a decrease of $\Delta A_{0}$ favors concavities (e.g. stomatocytic shapes), see Fig. 1. Consequently, the index of cell shape is expected to depend on the area difference, that is $I_{\mathrm{cs}}=I_{\mathrm{cs}}\left(\Delta A_{0}\right)$. For small area changes the latter dependence can be expanded in series:

$I_{\mathrm{cs}}=a_{1}+a_{2} \Delta A_{0}+\cdots$

where $a_{1}$ and $a_{2}$ are coefficients independent of $\Delta A_{0}$. The substitution of Eq. (6.1) into Eq. (6.2) shows that $I_{\mathrm{cs}}$ is expected to be a linear function of $\Delta \gamma$, which can be expressed in the form

$I_{\mathrm{cs}}=C\left(\Delta \gamma-\Delta \gamma_{0}\right)$
$C$ and $\Delta \gamma_{0}$ are coefficients, which should be independent of $\Delta \gamma$ and of the ionic strength. Our fits of the experimental data indicate that $C$ is independent also of osmolarity, whereas $\Delta \gamma_{0}$ increases with the decrease of osmolarity. Note that for $\Delta \gamma=\Delta \gamma_{0}$, Eq. (6.3) gives $I_{\mathrm{cs}}=0$ (discocyte shape), which means that $\Delta \gamma_{0}$ is the surface-pressure difference between the outer and inner leaflets for a discocyte.

\subsection{Procedure of data processing}

Eq. (6.3) lies at the roots of the procedure for data processing. For each given osmolarity, we plot $I_{\mathrm{cs}}$ versus $\Delta \gamma$ and process the data with a linear regression in accordance with Eq. (6.3). To do that, the values of $I_{\mathrm{cs}}$ are taken from the experiment (see the fourth column of Table 2 or 3 ), whereas $\Delta \gamma\left(I_{1}, I_{2}, \Delta \Phi\right)$ is calculated theoretically (using the equations in Sections 4 and 5) for each triad of values $\left(I_{1}, I_{2}\right.$, $\Delta \Phi)$, given in every row of Tables 2 and 3 .

To calculate $\Delta \gamma\left(I_{1}, I_{2}, \Delta \Phi\right)$, we have first to specify the values of the material parameters that enter the equations in Sections 4 and 5. For the dielectric constants of the aqueous phases and the hydrocarbon membrane core we use $\varepsilon_{\mathrm{w}}=$ 78.25 and $\varepsilon_{\mathrm{m}}=1.99$, which are the respective values for water and $n$-decane at the working temperature $25^{\circ} \mathrm{C}$. For the membrane thickness we use $2 h=5 \mathrm{~nm}$. As specified in Section 4.2, in Eq. (4.14) we substituted the values $\delta_{1}=$ $\delta_{2}=0.7 \mathrm{~nm}, \Gamma_{1}^{(1)}=0.25 \mathrm{~nm}^{-2}$ and $\Gamma_{1}^{(2)}=0.124 \mathrm{~nm}^{-2}$. The latter value is applicable only to native erythrocytes; the trypsin-treatment is expected to decrease the density of the ionizable groups at the outer membrane surface. For this reason, we introduced the dimensionless parameter

$\lambda=\frac{\left[\Gamma_{1}^{(2)}\right] \text { trypsin treatment }}{\Gamma_{1}^{(1)}}$

$\Gamma_{1}^{(1)}$ is supposed to be the same for native and trypsin-treated cells, while $\lambda$ is unknown.

Thus, we have three unknown quantities, $\lambda$ and the adsorption energies $\Delta \mu^{(1)}$ and $\Delta \mu^{(2)}$ in Eq. (4.14), which have been determined as adjustable parameters from the data fits, by means of the least squares method, that is by a numerical minimization of the function:

$\Psi\left(\Delta \mu^{(1)}, \Delta \mu^{(2)}, \lambda\right)=\sum_{j}\left[I_{\mathrm{cs}}^{(j)}-I_{\mathrm{cs}}^{\mathrm{th}}\left(\Delta \gamma^{(j)}\right)\right]^{2}$

where the index $j$ numbers the experimental points, which correspond to the rows of Tables 2 and $3 ; I_{\mathrm{cs}}^{(j)}$ are the experimental values of the cell-shape index, while $I_{\mathrm{cs}}^{\mathrm{th}}\left(\Delta \gamma^{(j)}\right)$ are the theoretical values of $I_{\mathrm{cs}}$, calculated by substituting $\Delta \gamma=\Delta \gamma^{(j)}$ in the linear regressions drawn in accordance with Eq. (6.3). The theoretical $\Delta \gamma^{(j)}=\Delta \gamma^{(j)}\left(I_{1}^{(j)}, I_{2}^{(j)}, \Delta \Phi^{(j)} ; \Delta \mu^{(1)}, \Delta \mu^{(2)}, \lambda\right)$, in its own turn, is calculated with the help of the expressions from Sections 4 and 5 as explained in Appendix A. In our computations, the summation in Eq. (6.5) was carried out over all experimental points in Tables 2 and 3, i.e. for all 
ionic strengths, osmolarities, for native and trypsin-treated cells, simultaneously. This was done to increase the statistics and to determine more reliable values of the adjustable parameters $\Delta \mu^{(1)}, \Delta \mu^{(2)}$ and $\lambda$ from the best fit. The latter corresponds to the minimum of $\Psi$ with respect to its three arguments, see Eq. (6.5).

The computations showed that there is a sharp minimum of $\Psi$ with respect to $\Delta \mu^{(2)}$ and $\lambda$. What concerns $\Delta \mu^{(1)}$, the function $\Psi$ initially decreases with the rise of $\Delta \mu^{(1)}$ for $0<\Delta \mu^{(1)}<8 k T$, but after that, for $\Delta \mu^{(1)}>8 k T$, the function $\Psi$ is practically constant. This means that for our experimental set of data, the parameter $\Delta \mu^{(1)}$ is $>8 k T$, but we are unable to determine its exact value using the specific data. In such a case, we have to assign some value to $\Delta \mu^{(1)}$ on the basis of physical considerations, and then to determine the other two adjustable parameters. Having in mind the discussion in Section 4.2, we specified $\Delta \mu^{(1)}=9 k T$; in other words, we assumed that we are dealing with a strong adsorption of counterions at the inner membrane surface. With this fixed value of $\Delta \mu^{(1)}$, we completed the numerical minimization and determined $\Delta \mu^{(2)}=2.13 k T$ and $\lambda=$ 0.335 for the best fit. As already mentioned, the numerical procedure is outlined in Appendix A.

\subsection{Parameter values determined from the best fit}

The obtained values of $\Delta \mu^{(2)}$ and $\lambda$ are reasonable. $\Delta \mu^{(2)}=2.13 k T$ is slightly greater than the adsorption energy of $\mathrm{Na}^{+}$ions at sulfate surface groups, $1.64 k T$ $[39,41,57]$. The difference might be due to the nature of the surface groups: sulfate versus sialic acid. In addition, a greater value of $\Delta \mu^{(2)}$ for our case may effectively account for the fact, that the outer phase contains not only $\mathrm{Na}^{+}$ions, but also $\mathrm{Mg}^{2+}$ ions.

$\lambda=0.335$ means that for the trypsin-treated cells $\Gamma_{1}^{(2)}=$ $0.0837 \mathrm{~nm}^{-2}$ (see Eq. (6.4)), which is about $67 \%$ of the value of $\Gamma_{1}^{(2)}$ for native erythrocytes. In other words, the trypsin-treatment has lead to a decrease of the glycocalyx charge with $33 \%$, which seems a reasonable value.

The standard deviation of the best fit is $\left[\Psi_{\min } /(N-\right.$ 1) $]^{1 / 2}=0.322$, where $N=26$ is the total number of experimental points in Tables 2 and 3 for osmolarities 290, 156 and $146 \mathrm{mOsm}$. This standard deviation seems reasonably small in view of the considerable scattering of the experimental $I_{\mathrm{cs}}$, see Figs. 4 and 5.

The values of the parameters $C$ and $\Delta \gamma_{0}$ in Eq. (6.3), corresponding to the best fit, are listed in Table 4. Because

Table 4

Parameter values in Eq. (6.3) corresponding to the best fit of the data

\begin{tabular}{llllll}
\hline $\begin{array}{l}\text { Osmolarity } \\
(\mathrm{mOsm})\end{array}$ & \multicolumn{2}{l}{ Native erythrocytes } & & \multicolumn{2}{l}{ Trypsin-treated erythrocytes } \\
\cline { 2 - 3 } & $C(\mathrm{~m} / \mathrm{mN})$ & $\Delta \gamma_{0}(\mathrm{Mn} / \mathrm{m})$ & & $C(\mathrm{~m} / \mathrm{mN})$ & $\Delta \gamma_{0}(\mathrm{mN} / \mathrm{m})$ \\
\hline 290 & 5.9 & 0.001 & & 8.5 & 0.100 \\
156 & 5.9 & 0.489 & & 8.5 & 0.462 \\
146 & 5.9 & 0.473 & & 8.5 & 0.493 \\
\hline
\end{tabular}

during the data processing we noticed that $C$ is independent of osmolarity, we included $C$ is constant in the computer program, which was thus simplified. On the other hand, the data in Table 4 indicate that $C$ is greater for the trypsin-treated cells. The tension difference for the dyscocites, $\Delta \gamma_{0}$, increases with the decrease of osmolarity; this tendency is better pronounced for the trypsin-treated cells. At $290 \mathrm{mOsm}$ we obtain $\Delta \gamma_{0} \approx 0$ for the native erythrocytes, which is in agreement with the fact that $290 \mathrm{mOsm}$ is the osmolarity at standard physiological conditions.

To visualize the agreement between theory and experiment, in Fig. 7 we have plotted the experimental cell-shape index, $I_{\mathrm{cs}}$, versus $\Delta \gamma-\Delta \gamma_{0}$. Thus, the data for all osmolarities can be shown in the same plot, despite the different $\Delta \gamma_{0}$. Fig. 7 indicates that $I_{\mathrm{cs}}$ really correlates with $\Delta \gamma$, in accordance with the linear dependence given by Eq. (6.3). For the native erythrocytes, the slope of the linear regression is $C=5.9 \pm 0.8(\mathrm{~m} / \mathrm{mN})$; the correlation coefficient is 0.907 . For the trypsin-treated erythrocytes, the slope of the
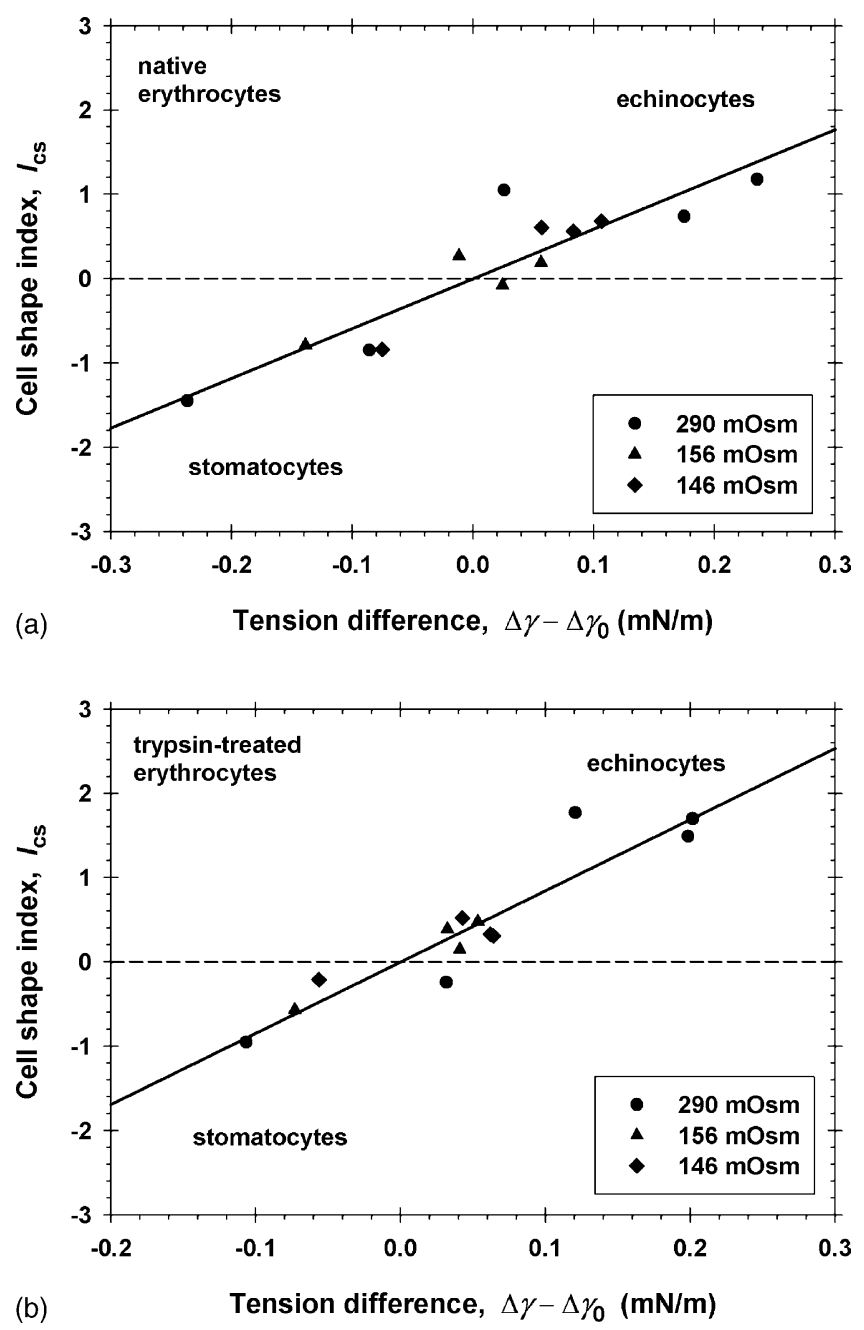

Fig. 7. Plot of the experimental cell shape index, $I_{\mathrm{cs}}$, vs. the calculated tension difference, $\Delta \gamma-\Delta \gamma_{0}$ : data for (a) native erythrocytes; (b) trypsin-treated erythrocytes. The lines represent regressions drawn in accordance with Eq. (6.3); see Table 4. 
linear regression is $C=8.5 \pm 0.9(\mathrm{~m} / \mathrm{mN})$; the correlation coefficient is 0.932 .

\subsection{Membrane charge and potential: results and discussion}

The results, reported below for the membrane electric properties, are calculated using equations from Sections 4 and 5, and parameter values corresponding to the best fit (Section 6.3).

The electric charge density at the two membrane surfaces is determined by the difference between the surface concentrations of the negative surface ionizable groups and of the positive adsorbed counterions, that is $\sigma_{\mathrm{s}, i}=-e\left(\Gamma_{1}^{(i)}-\Gamma_{2}^{(i)}\right), i=1,2$. The counterion adsorption $\Gamma_{2}^{(i)}$ increases with the ionic strength, while $\Gamma_{1}^{(i)}$ is constant. Consequently, the net surface charge decreases with the rise of the ionic strength. To visualize this tendency, in Fig. 8

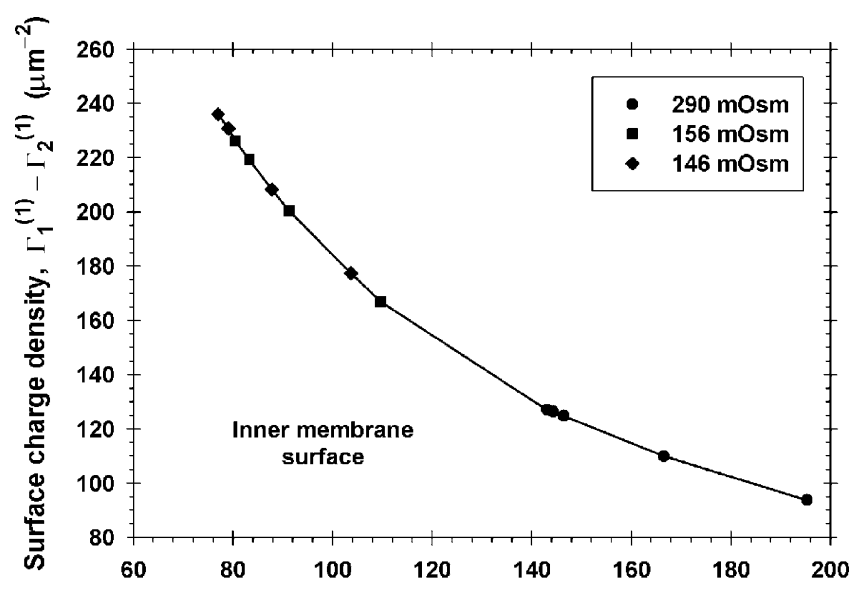

(a) lonic strength of the inner medium, $I_{1}(\mathrm{mM})$

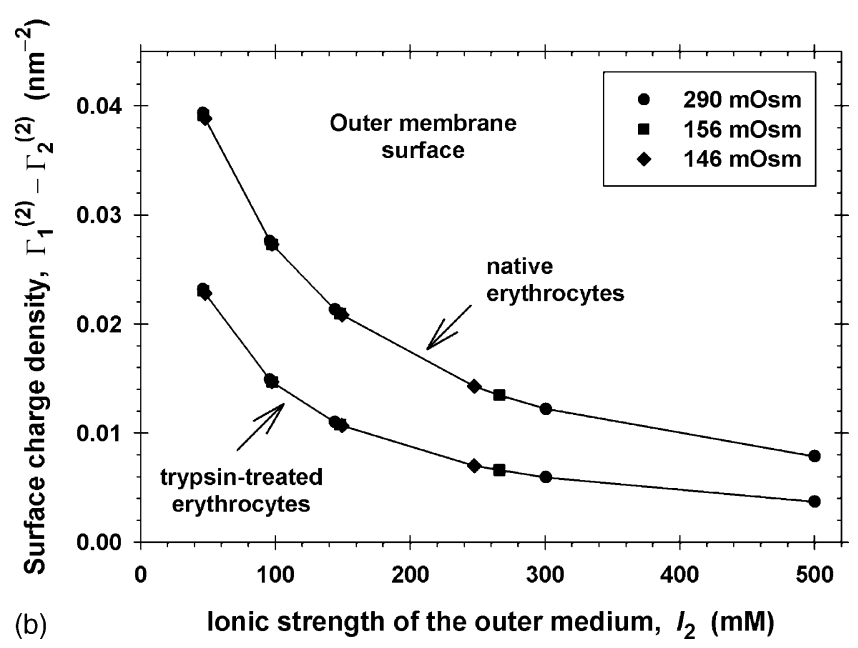

Fig. 8. Plots of the calculated net surface charge density of the erythrocyte membrane vs. the ionic strength of the adjacent phase: (a) the inner surface charge $\left(\Gamma_{1}^{(1)}-\Gamma_{2}^{(1)}\right)$ vs. $I_{1}$; (b) The outer surface charge $\left(\Gamma_{1}^{(2)}-\Gamma_{2}^{(2)}\right)$ vs. $I_{2}$. The density of the surface ionizable groups is fixed: $\Gamma_{1}^{(1)}=0.25 \mathrm{~nm}^{-2}$; $\Gamma_{1}^{(2)}=0.124$ and $0.0837 \mathrm{~nm}^{-2}$ for the native and trypsin-treated cells, respectively. we have plotted $\left(\Gamma_{1}^{(i)}-\Gamma_{2}^{(i)}\right)$ versus the ionic strength in the respective adjacent phase, $I_{i}$.

The calculated charge at the inner membrane surface (Fig. 8a) is the same for native and trypsin-treated cells. This result is in agreement with the fact that the trypsin-treatment modifies only the outer membrane surface. The calculated $\left(\Gamma_{1}^{(2)}-\Gamma_{2}^{(2)}\right)$ in Fig. 8b is pronouncedly smaller for the treated cells. This was to be expected, insofar as the trypsin is known to remove a part of the glycocalyx and to reduce the density of negative ionizable groups at the outer surface.

Qualitatively, the charge density on the inner and outer membrane surfaces decreases in a similar way with the rise of the ionic strength (Fig. 8). However, a comparison between Fig. 9a and b shows that the net charge density at the inner surface is about 100 times lower than on the outer surface. This considerable difference is due to the greater adsorption energy of the counterions at the inner surface:
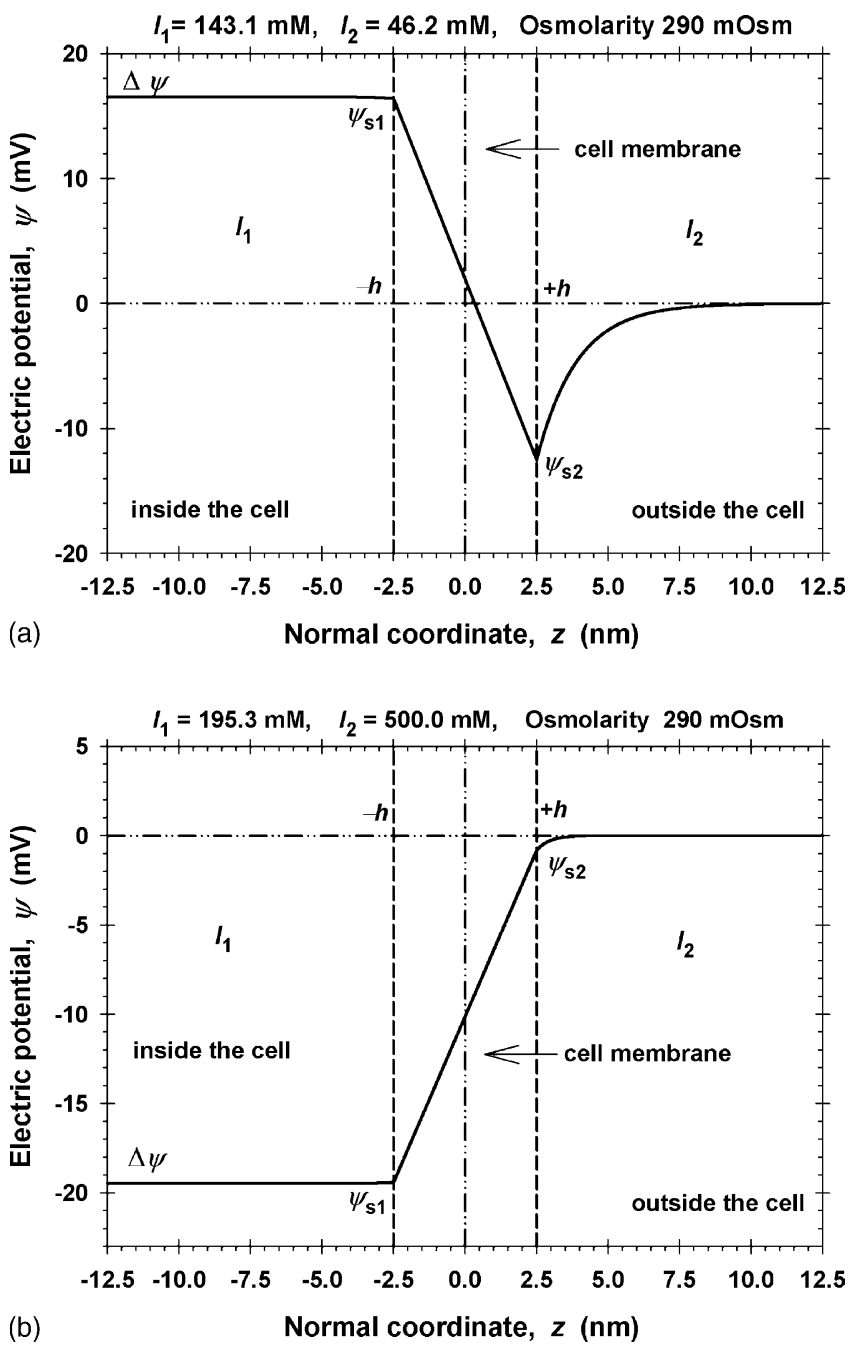

Fig. 9. Profile of the electric potential, $\psi(z)$, across the cell membrane, calculated for parameter values determined from the best fit of the data (Section 6.3). The ionic strengths correspond to experimental values from Table 2: (a) $I_{1}=143.1$ and $I_{2}=46.2 \mathrm{mM}$; (b) $I_{1}=195.3$ and $I_{2}=500 \mathrm{mM}$. In both cases, the osmolarity is $290 \mathrm{mOsm}$. The notations are as in Fig. 6. 
$\Delta \mu^{(1)} \approx 9 k T$ versus $\Delta \mu^{(2)}=2.13 k T$ at the outer surface (see above). Therefore, the counterion adsorption is greater at the inner surface, whose negatively charged groups are neutralized to a greater extent by the bound counterions. Nevertheless, the residual 100-200 charges per $\mu \mathrm{m}^{2}$ at the inner surface (Fig. 8a) still have a sufficiently high density, which can produce a considerable membrane-tension variation upon change of the ionic strength. To check that, we substituted $\left(\Gamma_{1}^{(1)}-\Gamma_{2}^{(1)}\right) \equiv 0$ in our computer program and tried to process the experimental data again; the result was that it is impossible to fit the data assuming zero electric charge at the inner membrane surface.

Another consequence from the low charge density at the inner surface is that the electric potential is practically uniform inside the cell. The inner surface and bulk potentials are approximately equal, $\Phi_{\mathrm{s} 1} \approx \Delta \Phi$, see Tables 2 and 3 . This is visualized in Fig. 9, where the computed potential distribution, $\psi(z)$, is shown for two experimental ionic strengths from Table 2. Although $\psi$ is independent of $z$ inside the cell, $\psi$ varies with the ionic strength because of the variation of $\Delta \psi$. For the considered range of ionic strengths and osmolarities, the potential of the outer membrane surface, $\Phi_{\mathrm{s} 2}$, is always negative and decreases by magnitude with the rise of the ionic strength (Tables 2 and 3; Fig. 9). The calculated surface potentials, $\Phi_{\mathrm{s} 1}$ and $\Phi_{\mathrm{s} 2}$, have been substituted in Eqs. (5.15)-(5.20) to determine the changes in the tensions of the membrane leaflets with the ionic strength.

\subsection{Membrane tensions: results and discussion}

Let us come back to the basic question about the physical reason for the increase of $\Delta \gamma$ with the outer ionic strength, $I_{2}$. In fact, this rise of $\Delta \gamma$ causes the increase of the area difference, $\Delta A_{0}$, leading to the stomatocyte-echinocyte transformation (see Section 6.1).
The tensions considered in this section are calculated by using parameter values corresponding to the best fit (Section 6.3). The last two columns of Tables 2 and 3 show the total membrane tension, $\gamma$, and the tension difference, $\Delta \gamma$, between the two leaflets. Firstly, one sees that the variations of the ionic strength and osmolarity lead to values of $\gamma$ and $\Delta \gamma$ on the order of $0.1 \mathrm{mN} / \mathrm{m}$. These are considerable tensions for an erythrocyte membrane, in view of the fact that the typical values for a relaxed membrane are on the order of $2 k_{\mathrm{c}} / R^{2} \approx 2.3 \times 10^{-5} \mathrm{mN} / \mathrm{m}$, where we have substituted $k_{\mathrm{c}}=1.8 \times 10^{-19} \mathrm{~J}$ for the bending elastic modulus [67], and $R=3.92 \mu \mathrm{m}$ for the average radius of a red blood cell [68]. The values of $\gamma$ decrease with the rise of the ionic strength (Tables 2 and 3 ), which means that the membrane, as a whole, becomes more flaccid. In contrast, $\Delta \gamma$ becomes greater with the ionic strength, which means that the stretching surface pressure of the outer leaflet increases relative to the surface pressure of the inner leaflet, see Eq. (5.23). The smallest and greatest values of $\Delta \gamma$ in Table $2, \Delta \gamma=-0.247$ and $+0.577 \mathrm{mN} / \mathrm{m}$, correspond to $\Delta A_{0} / A=-0.494$ and $+1.154 \%$; we have applied Eq. (6.1) with $K_{\mathrm{s}}=50 \mathrm{mN} / \mathrm{m}$. As order of magnitude, the latter values of $\Delta A_{0} / A$ belong to the range in which the formation of stomatocytes and echinocytes is reported in [33]. In other words, the predicted $\Delta \gamma$, stemming from the ionic strength variation, is sufficiently large to produce stomatocyte-echinocyte transformations.

Eq. (5.23) shows that $\Delta \gamma$ is a sum of two terms, which are due to the electric double layers and to the adsorption of counterions: $\Delta \gamma=\Delta \gamma_{\mathrm{el}}+\Delta \gamma_{\mathrm{ad}}$. The values of $\Delta \gamma_{\mathrm{el}}$ and $\Delta \gamma_{\text {ad }}$ are listed in Table 5 for native and trypsin-treated cells, at the same experimental $I_{2}$ as in Tables 2 and 3. The results in Table 5 are calculated using Eqs. (5.15), (5.16), (5.19), (5.20) and (5.24) from the values of the surface potentials

Table 5

Components of the membrane tension, $\gamma$, and of the tension difference between the two leaflets, $\Delta \gamma$, calculated for the values of $I_{2}$ in Tables 2 and 3 using Eqs. (5.15)-(5.24)

\begin{tabular}{|c|c|c|c|c|c|c|}
\hline \multirow[t]{2}{*}{$I_{2}(\mathrm{mM})$} & \multicolumn{3}{|c|}{ Native erythrocytes } & \multicolumn{3}{|c|}{ Trypsin-treated erythrocytes } \\
\hline & $\gamma_{1, \mathrm{ad}}(\mathrm{mN} / \mathrm{m})$ & $\Delta \gamma_{\mathrm{ad}}(\mathrm{mN} / \mathrm{m})$ & $\Delta \gamma_{\mathrm{el}}(\mathrm{mN} / \mathrm{m})$ & $\gamma_{1, \text { ad }}(\mathrm{mN} / \mathrm{m})$ & $\Delta \gamma_{\mathrm{ad}}(\mathrm{mN} / \mathrm{m})$ & $\Delta \gamma_{\mathrm{el}}(\mathrm{mN} / \mathrm{m})$ \\
\hline \multicolumn{7}{|c|}{ Cells at osmolarity $290 \mathrm{mOsm}$} \\
\hline 46.24 & 0.0524 & -0.2779 & 0.0324 & 0.0529 & -0.2186 & 0.0117 \\
\hline 95.79 & 0.0473 & -0.1022 & 0.0075 & 0.0475 & -0.0710 & 0.0023 \\
\hline 144.2 & 0.0339 & 0.0163 & 0.0006 & 0.0339 & 0.0201 & 0.0002 \\
\hline 300.5 & -0.0966 & 0.1706 & -0.0046 & -0.0967 & 0.1026 & -0.0013 \\
\hline 500.0 & -0.2601 & 0.2322 & -0.0057 & -0.2604 & 0.0997 & -0.0015 \\
\hline \multicolumn{7}{|c|}{ Cells at osmolarity $156 \mathrm{mOsm}$} \\
\hline 46.62 & 0.6453 & 0.3177 & 0.0325 & 0.6461 & 0.3769 & 0.0118 \\
\hline 97.54 & 0.6136 & 0.4702 & 0.0073 & 0.6139 & 0.5003 & 0.0023 \\
\hline 148.0 & 0.5215 & 0.5132 & 0.0003 & 0.5216 & 0.5150 & 0.0001 \\
\hline 266.1 & 0.3329 & 0.5495 & -0.0041 & 0.3327 & 0.4951 & -0.0011 \\
\hline \multicolumn{7}{|c|}{ Cells at osmolarity $146 \mathrm{mOsm}$} \\
\hline 47.58 & 0.6899 & 0.3662 & 0.0314 & 0.6908 & 0.4250 & 0.0114 \\
\hline 97.26 & 0.6667 & 0.5223 & 0.0073 & 0.6670 & 0.5526 & 0.0023 \\
\hline 149.3 & 0.5611 & 0.5557 & 0.0002 & 0.5611 & 0.5568 & 0.0001 \\
\hline 247.5 & 0.3960 & 0.5828 & -0.0038 & 0.3959 & 0.5364 & -0.0011 \\
\hline
\end{tabular}




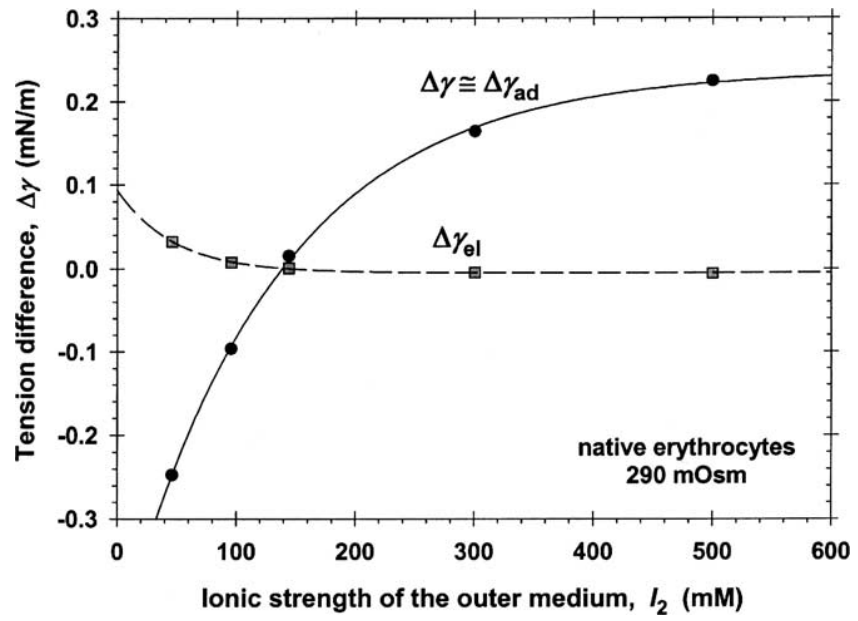

Fig. 10. Plot of the tension differences due to the counterion adsorption and electric double layer, $\Delta \gamma_{\mathrm{ad}}$ and $\Delta \gamma_{\mathrm{el}}$ vs. the ionic strength of the outer medium, $I_{2}$. The points are numerical results from Table 5 (native erythrocytes at $290 \mathrm{mOsm}$ ), calculated using Eqs. (5.15)-(5.24) for parameter values corresponding to the best fit (Section 6.3). As $\left|\Delta \gamma_{\text {ad }}\right| \gg\left|\Delta \gamma_{\mathrm{el}}\right|$, it turns out that $\Delta \gamma \approx \Delta \gamma_{\text {ad }}$.

(Section 6.4). One sees that $\Delta \gamma_{\mathrm{el}}$ decreases, whereas $\Delta \gamma_{\mathrm{ad}}$ increases with the ionic strength. This behavior of $\Delta \gamma_{\mathrm{el}}$, which is due to the screening of the repulsion between electric charges at the outer membrane surface by the electrolyte, could be considered as a contradiction with the bilayer-couple hypothesis [7]. In the present article, we take into acount the effect of $\Delta \gamma_{\mathrm{ad}}$, in addition to $\Delta \gamma_{\mathrm{el}}$. It turns out that $\left|\Delta \gamma_{\mathrm{el}}\right| \ll\left|\Delta \gamma_{\mathrm{ad}}\right|$, and that $\Delta \gamma$ is completely dominated by $\Delta \gamma_{\mathrm{ad}}$, which varies in the opposite to $\Delta \gamma_{\mathrm{el}}$ direction (Table 5). This is illustrated in Fig. 10. In other words, there is no contradiction between the bilayer-couple hypothesis and the electric double layer theory.

From the data in Table 5 one can calculate also $\gamma_{2, \text { ad }}=$ $\gamma_{1, \text { ad }}-\Delta \gamma_{\text {ad }}$. It is remarkable that both $\gamma_{1, \text { ad }}$ and $\gamma_{2, \text { ad }}$ decrease with the rise of $I_{2}$, however $\gamma_{2}$,ad decreases faster, which leads to increasing of their difference, $\Delta \gamma_{\mathrm{ad}}$. In other words, the increase of $\Delta \gamma_{\text {ad }}$ with $I_{2}$ results from a fine difference between similar tendencies in the behavior of the two membrane leaflets. The slower variation of $\gamma_{1 \text {,ad }}$, relative to $\gamma_{2, \text { ad }}$, can be attributed to the fact that (i) the inner ionic strength, $I_{1}$, exhibits a weaker vatiation than the outer one, $I_{2}$, see Table 2; (ii) the adsorption of counterions at the inner leaflet (unlike that at the outer leaflet) is very close to a complete saturation (cf. Fig. 9a and b, where $\Gamma_{1}^{(1)}-$ $\Gamma_{2}^{(1)} \ll \Gamma_{1}^{(2)}-\Gamma_{2}^{(2)}$ ), and correspondingly, the increase of counterion adsorption with the ionic strength should be slower for the inner leaflet, in comparison with the outer one.

Our calculations showed that $\gamma_{\mathrm{m}, \mathrm{el}}$, that is the tension due to the variation of the electric potential across the membrane, see Eq. (5.17), turns out to be negligible in comparison with $\gamma_{1, \text { el }}$ and $\gamma_{2, \mathrm{el}}$. Hence, the contribution of $\gamma_{\mathrm{m} \text {,el }}$ to the total membrane tension, $\gamma$, is immaterial; see Eq. (5.14).

In the framework of our model, the osmolarity affects the erythrocyte shape (i) indirectly, through the values of $I_{1}$ and
$\Delta \Phi$ computed by means of the integrated red cell model $[45,46]$, see Tables 2 and 3; and (ii) directly, through the tension difference for discocyte, $\Delta \gamma_{0}$; thus, when the osmolarity decreases, $\Delta \gamma_{0}$ increases (Table 4 ) and, according to Eq. (6.3), $I_{\mathrm{cs}}$ decreases, that is the cell shape shifts toward stomatocytes.

What concerns the effect of $\mathrm{pH}$, see [8-12], it could be attributed to a competitive binding of $\mathrm{H}^{+}$and the other cations $\left(\mathrm{Na}^{+}, \mathrm{K}^{+}\right.$, etc. $)$at the negatively charged residues on the two membrane surfaces. In particular, the $\mathrm{pH}$-dependent association-dissociation of the - $\mathrm{COOH}$ groups at the membrane surfaces is also a kind of $\mathrm{H}^{+}$binding. An additional effect could be produced by the attachment of $\mathrm{OH}^{-}$ions to the membrane, as it is known that the hydroxyl ions exhibit a tendency to adsorb at the boundary water-hydrocarbon [69]. The influence of the $\mathrm{H}^{+}$and $\mathrm{OH}^{-}$ions on the membrane tension can be also described by means of equations similar to Eq. (5.10), however, a multicomponent ionic adsorption should be considered, as in [59].

In the same way, one could take into account the effect of amphiphiles, which also influence the cell shape [13-18]. It is an inherent property of the amphiphiles to adsorb at phase boundaries, including cell membranes. Again, one could employ a version of the Szyszkowski-Langmuir Eq. (5.10) to quantify the effect of the amphiphiles on the tensions of the membrane leaflets. Depending on the type of the amphiphile and its interaction with the membrane, the amphiphile could adsorb either with its hydrophobic tail, or with its hydrophilic headgroup, on the membrane surface.

Finally, the observed correlation between the conformational changes of the band 3 protein and the erythrocyte shape [27-30], might also be related with the proposed physicochemical model. For example, if the amphiphiles adsorb on band 3 and inhibit the anion transport [30], this can affect the membrane tension in two ways: (i) a direct decrease of the membrane tension upon adsorption, see Eq. (5.10); (ii) an indirect effect due to inhibiting of the anion transport, which will alter the values of $I_{1}$ and $\Delta \Phi$ (see Tables 2 and 3), and will influence $\Phi_{\mathrm{s} 1}, \Phi_{\mathrm{s} 2}$, and $\Delta \gamma$, see Eqs. (4.15), (4.16) and (5.15)-(5.24).

\section{Summary and conclusions}

In the present article, we try to understand the way in which the increase of the outer medium's ionic strength leads to stomatocyte-echinocyte transformations of human red blood cells. With this end in view, we determined experimentally the index of cell shape at various ionic strengths and osmolarities for native erythrocytes, and for trypsin-treated ones, see Figs. 2-5. For every composition of the outer phase, we calculated the ionic strength inside the cells and the transmembrane electric potential (Tables 2 and 3 ) by using an available theoretical model $[45,46]$. The latter accounts for both the passive and active transport of ions across the membrane. Next, we described theoretically 
the electric double layers formed on both sides of the cell membrane (Section 4), and derived expressions for calculating the tensions of the outer and inner membrane leaflets (Section 5). Taking into account the dependence of the cell-shape index on the tension difference between the two leaflets, Eq. (6.3), we fitted the experimental data with the constructed theoretical model and determined, as adjustable parameters, the counterion adsorption energies at the inner and outer membrane surface ( 9 and $2 k T$ per ion) and the reduction of the glycocalyx charge by the trypsin-treatment (with 33\%), see Section 6.3.

The model, which agrees well with the experimental data (Fig. 7), indicates two reasons for the appearance of a tension difference between the two leaflets: (i) different electric double layers on the two sides of the membrane (Fig. 9); and (ii) different adsorption of counterions at the two membrane surfaces (Fig. 8). It turns out that the latter effect is predominant (Fig. 10; Table 5). Thus, the following picture of the cell-shape transformation emerges from our study. With the rise of the ionic strength, the counterion adsorption increases stronger at the outer leaflet; this induces a greater stretching surface pressure in this leaflet, whose area expands relative to the inner one. The latter expansion produces a tendency to form convex structures on the cell surface (e.g. echinocytic spicules). Conversely, a decrease of the ionic strength favors concavities (e.g. stomatocytic shapes). Hence, there is no contradiction between the bilayer-couple hypothesis and the electric double layer theory, if the latter is upgraded to account for the contribution of the counterion adsorption to the membrane tension. The developed quantitative model can be applied to predict the index of cell shape, along the axis "stomatocyte-discocyte-echinocyte," for various ionic strengths and osmolarities of the outer medium.

\section{Acknowledgements}

The support from the Franco-Bulgarian Laboratory "Vesicles and Membranes", coordinated by Professor Philippe Méléard, is gratefully acknowledged. The authors are indebted to Miss. Svetlana Nikolova for her assistance in the experiments.

\section{Appendix A. Principles of the computational procedure}

1. The input parameters are the data for $I_{2}, I_{1}, \Delta \Phi$ and $I_{\mathrm{cs}}$ in the first four columns of Tables 2 and 3 . We use also the following parameter values: $\delta_{1}=\delta_{2}=0.7 \mathrm{~nm}$; $2 h=5 \mathrm{~nm} ; \varepsilon_{\mathrm{w}}=78.25 ; \varepsilon_{\mathrm{m}}=1.99 ; \Gamma_{1}^{(1)}=0.25 \mathrm{~nm}^{-2}$. For native cells $\Gamma_{1}^{(2)}=0.124 \mathrm{~nm}^{-2}$; for trypsin-treated cells we substitute $\Gamma_{1}^{(2)}=\lambda \Gamma_{1}^{(1)}=\lambda \times 0.25 \mathrm{~nm}^{-2} ; \lambda$ is determined as an adjustable parameter from the best fit of the data.
2. $\kappa_{1}$ and $\kappa_{2}$ are calculated from Eq. (4.2), which is written in terms of the CGSE system; therefore one one must substitute $k=1.38 \times 10^{-16} \mathrm{erg} / \mathrm{K}, \quad e=4.80 \times 10^{-10}$ CGSE units; $I_{i}$ should be in $\mathrm{cm}^{-3}$ and then $\kappa_{i}$ is obtained in $\mathrm{cm}^{-1}(i=1,2)$.

3. Trial values are given to $\Delta \mu^{(1)}, \Delta \mu^{(2)}$ and $\lambda$. Next, $K_{1}$ and $K_{2}$ are calculated from Eq. (4.14).

4. $\Phi_{\mathrm{s} 1}$ and $\Phi_{\mathrm{s} 2}$ are determined from Eqs. (4.15) and (4.16) as explained in Section 4.3; the system of units is as in point 2. Then, $\Gamma_{2}^{(1)}$ and $\Gamma_{2}^{(2)}$ can be computed from Eqs. (4.10) and (4.11).

5. $\gamma_{1, \mathrm{el}}, \gamma_{2, \mathrm{el}}, \gamma_{1, \text { ad }}$ and $\gamma_{2, \text { ad }}$ are calculated from Eqs. (5.15), (5.16), (5.19) and (5.20). Next, $\Delta \gamma_{\mathrm{ad}}$ and $\Delta \gamma_{\mathrm{el}}$ are computed from Eq. (5.24), and finally, $\Delta \gamma=\Delta \gamma_{\mathrm{ad}}+\Delta \gamma_{\mathrm{el}}$.

6. The obtained values of $\Delta \gamma$ will be denoted by $\Delta \gamma^{(\mathrm{n})}\left(\Omega^{(i)}, I_{2}^{(j)}\right)$ and $\Delta \gamma^{(\mathrm{t})}\left(\Omega^{(i)}, I_{2}^{(j)}\right)$, where the superscripts '(n)' and '(t)' refer to native and trypsin-treated cells; the indices ' $i$ ' and ' $j$ ' number the values of the outer ionic strength, $I_{2}$ and of the osmolarity: $\Omega^{(1)}=290$, $\Omega^{(2)}=156$ and $\Omega^{(3)}=146$ (mOsm) are the experimental osmolarities in our case. The functions $I_{1}\left(I_{2}\right)$ and $\Delta \Phi\left(I_{2}\right)$ are tabulated in Tables 2 and 3, where the experimental values of the cell-shape index, $I_{\mathrm{cs}}^{(i, j ; y)}$, are also given for each osmolarity $(i)$, ionic strength $(j)$, and type of cell $(y=n, t)$. Likewise, it is convenient to introduce the notation $\Delta \gamma^{(i, j ; y)} \equiv \Delta \gamma^{(y)}\left(\Omega^{(i)}, I_{2}^{(j)}\right)$.

7. For each given $\Omega^{(i)}$ and type of cells (native or trypsin-treated, $y=n, t)$ the data for $I_{\mathrm{cs}}^{(i, j ; y)}$ versus $\Delta \gamma^{(i, j ; y)}(j=1,2, \ldots)$ have to be fitted with a linear regression, in accordance with Eq. (6.3). The slope and intercept of each linear regression, characterized by the coefficients $C$ and $\Delta \gamma_{0}$ in Eq. (6.3), are determined by explicit expressions, originating from the least squares method. The expressions for $C$, which depends on the type of cell, are:

$C^{(y)}=\frac{A^{(y)}}{B^{(y)}} \quad(y=n, t)$

where

$A^{(y)}=\sum_{i=1}^{3} \sum_{j=1}^{N^{(i, y)}}\left[I_{\mathrm{cs}}^{(i, j ; y)}-\left\langle I_{\mathrm{cs}}^{(i ; y)}\right\rangle\right]\left[\Delta \gamma^{(i, j ; y)}-\left\langle\Delta \gamma^{(i ; y)}\right\rangle\right]$

$B^{(y)}=\sum_{i=1}^{3} \sum_{j=1}^{N^{(i ; y)}}\left[\Delta \gamma^{(i, j ; y)}-\left\langle\Delta \gamma^{(i ; y)}\right\rangle\right]^{2}$

$$
\left\langle I_{\mathrm{cs}}^{(i ; y)}\right\rangle \equiv \frac{1}{N^{(i ; y)}} \sum_{j=1}^{N^{(i ; y)}} I_{\mathrm{cs}}^{(i, j ; y)}
$$

$\left\langle\Delta \gamma^{(i ; y)}\right\rangle \equiv \frac{1}{N^{(i ; y)}} \sum_{j=1}^{N^{(i ; y)}} \Delta \gamma^{(i, j ; y)}$ 
$N^{(i, y)}$ denotes the number of experimental points for a given osmolarity and type of cells. Analogously, for $\Delta \gamma_{0}$, which depends on both osmolarity and type of cells, the least-squares method gives the expression:

$$
\begin{aligned}
& \Delta \gamma_{0}^{(i ; y)}=\left\langle\Delta \gamma^{(i ; y)}\right\rangle-\frac{1}{C^{(y)}}\left\langle I_{\mathrm{cs}}^{(i ; y)}\right\rangle \\
& \quad(i=1,2,3 ; y=n, t)
\end{aligned}
$$

8. For each osmolarity and type of cells, from Eq. (6.3) we calculate the theoretical value of the cell-shape index:

$I_{\mathrm{cs}, \mathrm{th}}^{(i, j ; y)}=C^{(y)}\left(\Delta \gamma^{(i, j ; y)}-\Delta \gamma_{0}^{(i ; y)}\right)$

Note that $I_{\mathrm{cs}, \mathrm{th}}^{(i, j ; y)}$ depends on the adjustable parameters $\Delta \mu^{(1)}, \Delta \mu^{(2)}$ and $\lambda$.

9. Finally, we minimize numerically the dispersion

$$
\Psi\left(\Delta \mu^{(1)}, \Delta \mu^{(2)}, \lambda\right)=\sum_{y=n, t} \sum_{i=1}^{3} \sum_{j=1}^{N^{(i ; y)}}\left(I_{\mathrm{cs}}^{(i, j ; y)}-I_{\mathrm{cs}, \mathrm{th}}^{(i, j ; y)}\right)^{2}
$$

by variation of the parameters $\Delta \mu^{(1)}, \Delta \mu^{(2)}$ and $\lambda$. Thus, we determine the values of the latter parameters, which correspond to the best fit, i.e. to the minimum of $\Psi$. As already mentioned, it turned out that for our set of data, $\Psi$ has no minimum with respect to $\Delta \mu^{(1)}$. For this reason, on the basis of physical considerations, we substituted $\Delta \mu^{(1)}=9 k T$, and then the minimization of $\Psi$ was carried out with respect to $\Delta \mu^{(2)}$ and $\lambda$, see Section 6.2 for details.

\section{References}

[1] B. Deuticke, Transformation and restoration of biconcave shape of human erythrocytes induced by amphiphilic agents and changes of ionic environment, Biochim. Biophys. Acta 163 (1968) 494-500.

[2] L. Backman, Shape control in human red cell, J. Cell. Sci. 80 (1968) 281-298.

[3] A. Herrmann, P. Müller, R. Glaser, Shape transformation of erythrocyte ghosts depends on ion concentrations, Biosci. Rep. 5 (1985) 417-423.

[4] R. Glaser, T. Fujii, P. Müller, E. Tamura, A. Herrmann, Erythrocyte shape dynamics: influence of electrolyte conditions and membrane potential, Biomed. Biochim. Acta 46 (1987) 327-333.

[5] R. Glaser, C. Gengnagel, J. Donath, Membrane electric field and erythrocyte shape, Stud. Biophys. 127 (1988) 201-206.

[6] A. Elgsaeter, A. Mikkelsen, Shapes and shape changes in vitro in normal red blood cell, Biochem. Biophys. Acta 1071 (1991) 272290.

[7] M. Rasia, A. Bollini, Red blood cell shape as a function of medium's ionic strength and pH, Biochem. Biophys. Acta 1372 (1998) 198204.

[8] M. Gedde, E. Yang, W. Huestis, Shape response of human erythrocytes to altered cell pH, Blood 86 (1995) 1595-1599.

[9] M.M. Gedde, D.K. Davis, W.H. Huestis, Cytoplasmic pH and human erythrocyte shape, Biophys. J. 72 (1997) 1234-1246.

[10] J. Libera, T. Pomorski, P. Müller, A. Herrmann, Influence of $\mathrm{pH}$ on phospholipid redistribution in human erythrocyte membrane, Blood 90 (1997) 1684-1693.
[11] M.M. Gedde, W.H. Huestis, Membrane potential and human erythrocyte shape, Biophys. J. 72 (1997) 1220-1233.

[12] J. Gimsa, A possible molecular mechanism governing human erythrocyte shape, Biophys. J. 75 (1998) 568-569.

[13] G. Brecher, M. Bessis, Present status of spiculated red cells and their relationship to the discocyte-echinocyte transformation: a critical review, Blood 40 (1972) 333-344.

[14] M. Bessis, Red cell shapes: an illustrated classification and its rationale, in: M. Bessis, R.I. Wed, P.F. LeBlond (Eds.), Red Cell Shapes, Springer, New York, 1973, pp. 1-23.

[15] M.P. Sheetz, S.J. Singer, Biological membranes as bilayer couples. A mechanism of drug-erythrocyte interactions, Proc. Natl. Acad. Sci. U.S.A. 71 (1974) 4457-4461.

[16] B. Isomaa, H. Hägerstrand, G. Paatero, Shape transformations induced by amphiphiles in erythrocytes, Biochim. Biophys. Acta 899 (1987) 93-103.

[17] R. Glaser, C. Gengnagel, J. Donath, The influence of valinomycin induced membrane potential on erythrocyte shape, Biomed. Biochim. Acta 50 (1991) 869-877.

[18] A. Iglič, V. Kralj-Iglič, H. Hägerstrand, Amphiphile induced echinocyte-sphero-echinocyte transformation of red blood cell shape, Eur. Biophys. J. 27 (1998) 335-339.

[19] R. Glaser, The shape of red blood cells as a function of membrane potential and temperature, J. Membr. Biol. 51 (1979) 217-228.

[20] R. Glaser, J. Donath, Temperature and transmembrane potential dependence of shape transformations of human erythrocytes, Biochem. Bioenerg. 27 (1992) 429-440.

[21] R. Glaser, Echinocyte formation induced by potential changes of human red blood cells, J. Membr. Biol. 66 (1982) 79-85.

[22] E.M. Bifano, T.S. Novak, J.C. Freedman, Relationship between the shape and the membrane potential of human red blood cells, J. Membr. Biol. 82 (1984) 1-13.

[23] P. Müller, A. Herrmann, R. Glaser, Further evidence for a membrane potential shape transformation of the human erythrocyte membrane, Biosci. Rep. 6 (1986) 999-1006.

[24] R. Glaser, Mechanisms of electromechanical coupling in membranes demonstrated by transmembrane potential-dependent shape transformations of human erythrocytes, Bioelectrochem. Bioenerg. 30 (1993) 103-109.

[25] R. Glaser, Does the Transmembrane potential or the intracellular pH control the shape of human erythrocytes? Biophys. J. 75 (1998) 569-570.

[26] R. Mukhopadhyay, G.H.W. Lim, M. Wortis, Echinocyte shapes: bending, stretching and shear determine spicule shape and spacing, Biophys. J. 82 (2002) 1756-1772.

[27] J. Gimsa, Red cell echinocytogenesis is correlated to the recruitment of external band-3 conformations, Bioelectrochem. Bioenerg. 38 (1995) 99-103.

[28] J. Gimsa, Ch. Ried, Do band 3 protein conformational changes mediate shape changes of human erythrocytes? Mol. Membr. Biol. 12 (1995) 247-254.

[29] P. Wong, Mechanism of control of erythrocyte shape: a possible relationship to band 3, J. Theor. Biol. 171 (1994) 197-205.

[30] P. Wong, A basis of echinocytosis and stomatocytosis in the discsphere transformations of the erythrocyte, J. Theor. Biol. 196 (1999) 343-361.

[31] E. Evans, Bending resistance and chemically induced moments in membrane bilayers, Biophys. J. 14 (1974) 923-931.

[32] A.G. Petrov, The Lyotropic State of Matter: Molecular Physics and Living Matter Physics, Gordon \& Breach, Amsterdam, 1999.

[33] G.H.W. Lim, M. Wortis, R. Mukhopadhyay, Stomatocyte-discocyteechinocyte sequence of the human red blood cell: evidence for the bilayer-couple hypothesis from membrane mechanics, Proc. Natl. Acad. Sci. U.S.A. 99 (2002) 16766-16769.

[34] Y. Lange, A. Gough, T. Steck, Role of the bilayer in the shape of the isolated erythrocyte membrane, J. Membr. Biol. 69 (1982) 113-123. 
[35] R. Grebe, H. Wolff, H. Schmid-Schönbein, Influence of red cell surface charge on red cell curvature, Pflügers Arch. 413 (1988) 77-82.

[36] J.Th.G. Overbeek, Electrochemistry of the double layer, in: H.R. Kruyt (Ed.), Colloid Science, vol. 1, Elsevier, Amsterdam, 1953.

[37] J.F. Rathman, J.F. Scamehorn, Counterion binding on mixed micelles, J. Phys. Chem. 88 (1984) 5807-5816.

[38] J. Clint, Surfactant Aggregation, Chapman \& Hall, London, 1992.

[39] R.G. Alargova, K.D. Danov, P.A. Kralchevsky, G. Broze, A. Mehreteab, Growth of giant rodlike micelles of ionic surfactant in the presence of $\mathrm{Al}^{3+}$ counterions, Langmuir 14 (1998) 4036-4049.

[40] V.V. Kalinin, C.J. Radke, An ion-binding model for ionic surfactant adsorption at aqueous-fluid interfaces, Colloids Surf. A 114 (1996) 337-350.

[41] P.A. Kralchevsky, K.D. Danov, G. Broze, A. Mehreteab, Thermodynamics of ionic surfactant adsorption with account for the counterion binding: effect of salts of various valency, Langmuir 15 (1999) 2351-2365.

[42] A.J. Prosser, E.I. Franses, Adsorption and surface tension of ionic surfactants at the air-water interface: review and evaluation of equilibrium models, Colloids Surf. A 178 (2001) 1-40.

[43] E. Donath, D. Gingell, A sharp cell surface conformational transition at low ionic strength changes the nature of the adhesion of enzymetreated red blood cells to a hydrocarbon interface, J. Cell Sci. 63 (1983) 113-124.

[44] K.D. Tachev, J.K. Angarska, K.D. Danov, P.A. Kralchevsky, Erythrocyte attachment to substrates: determination of membrane tension and adhesion energy, Colloids Surf. B: Biointerfaces 19 (2000) 61-80.

[45] V.L. Lew, R.M. Bookchin, Volume, $\mathrm{pH}$ and ion content regulation in human red cells: analysis of transient behavior with an integrated model, J. Membr. Biol. 92 (1986) 57-74.

[46] The "Red cell model computer programme" is presently available at the website of the Dr. V.L. Lew's Cell Physiology Laboratory, Department of Physiology, University of Cambridge, UK.

[47] S.L. Keller, W.H. Pitcher III, W.H. Huestis, H.M. McConnell, Red blood cell lipids form immiscible liquids, Phys. Rev. Lett. 81 (1998) 5019-5022.

[48] E. Donath, A. Budde, E. Knippel, H. Bäumler, "Hairy surface layer" concept of electrophoresis combined with local fixed surface charge density isotherms: application to human erythrocyte electrophoretic fingerprinting, Langmuir 12 (1996) 4832-4839.

[49] B.V. Derjaguin, Theory of Stability of Colloids and Thin Liquid Films, Plenum Press, New York, 1989.

[50] J.N. Israelachvili, Intermolecular and Surface Forces, Academic Press, London, 1992.

[51] P.A. Kralchevsky, K. Nagayama, Particles at Fluid Interfaces and Membranes, Elsevier, Amsterdam, 2001.

[52] J.C. Freedman, J.F. Hoffman, Ionic and osmotic equilibria of human red blood cells treated with nystatin, J. Gen. Physiol. 74 (1979) 157-185.
[53] I.E. Tamm, Fundamentals of Electricity Theory, Nauka, Moscow, 1989 (in Russian).

[54] O. Stern, Zur Theorie der Elektrischen Doppelschicht, Z. Elektrochem. 30 (1924) 508-516.

[55] J.T. Davies, E.K. Rideal, Interfacial Phenomena, Academic Press, London, 1963.

[56] E.D. Shchukin, A.V. Pertsov, E.A. Amelina, Colloid Chemistry, Moscow University Press, Moscow, 1982 (in Russian).

[57] V.L. Kolev, K.D. Danov, P.A. Kralchevsky, G. Broze, A. Mehreteab, Comparison of the van der Waals and Frumkin adsorption isotherms for sodium dodecyl sulfate at various salt concentrations, Langmuir 18 (2002) 9106-9109.

[58] P.A. Kralchevsky, K.D. Danov, V.L. Kolev, G. Broze, A. Mehreteab, Effect of nonionic admixtures on the adsorption of ionic surfactants at fluid interfaces. 1. Sodium dodecyl sulfate and dodecanol, Langmuir 19 (2003) 5004-5018.

[59] K.D. Danov, S.D. Kralchevska, P.A. Kralchevsky, G. Broze, A Mehreteab, Effect of nonionic admixtures on the adsorption of ionic surfactants at fluid interfaces. 2. Sodium dodecylbenzene sulfonate and dodecylbenzene, Langmuir 19 (2003) 5019-5030.

[60] K.S. Krasnov, Molecules and Chemical Bond, High School Publishing House, Moscow, 1984 (in Russian).

[61] S. Hachisu, Equation of state of ionized monolayers, J. Colloid Interface Sci. 33 (1970) 445-454.

[62] G. Bakker, Kapillarität und Oberflächenspannung, in: Handbuch der Experimental-physik, Band 6, Akademische Verlagsgesellschaft, Leipzig, 1928.

[63] S. Ono, S. Kondo, Molecular theory of surface tension in liquids, in: S. Flügge (Ed.), Handbook of Physics, vol. 10, Springer, Berlin, 1960, p. 134.

[64] L.D. Landau, E.M. Lifshitz, Electrodynamics of Continuous Medium, Pergamon Press, Oxford, 1960.

[65] A.W. Adamson, A.P. Gast, Physical Chemistry of Surfaces, sixth ed., Wiley/Interscience, New York, 1997.

[66] P.A. Kralchevsky, K.D. Danov, N.D. Denkov, Chemical physics of colloid systems and interfaces, in: K.S. Birdi (Ed.), Handbook of Surface and Colloid Chemistry, second ed., CRC Press, Boca Raton, FL, 2002 (Chapter 5).

[67] L. Fernandez-Puente, I. Bivas, M.D. Mitov, P. Méléard, Temperature and chain-length effects on bending elasticity of phosphatidylcholine bilayers, Europhys. Lett. 28 (1994) 181-186.

[68] R. Georgieva, B. Neu, V.M. Shilov, E. Knippel, A. Budde, R. Latza, E. Donath, H. Kiesewetter, H. Bäumler, Low frequency electrorotation of fixed red blood cells, Biophys. J. 74 (1998) 2114 2120.

[69] K.G. Marinova, R.G. Alargova, N.D. Denkov, O.D. Velev, D.N. Petsev, I.B. Ivanov, R.P. Borwankar, Charging of oil-water interfaces due to spontaneous adsorption of hydroxyl ions, Langmuir 12 (1996) 2045-2051. 